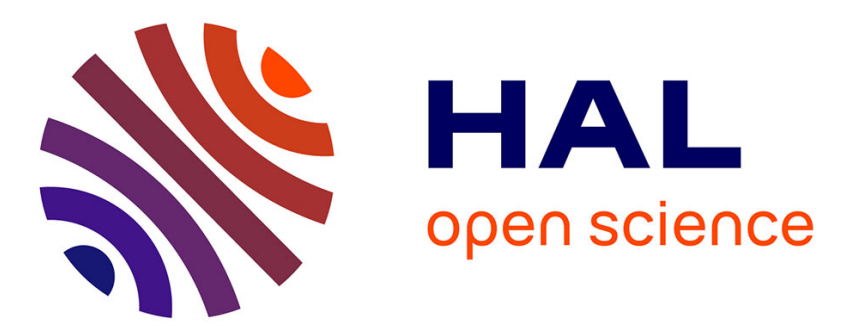

\title{
Variational formulation of micropolar elasticity using 3D hexahedral finite-element interpolation with incompatible modes
}

\author{
Sara Grbčić, Adnan Ibrahimbegović, Gordan Jelenić
}

\section{- To cite this version:}

Sara Grbčić, Adnan Ibrahimbegović, Gordan Jelenić. Variational formulation of micropolar elasticity using 3D hexahedral finite-element interpolation with incompatible modes. Computers \& Structures, 2018, 205, pp.1-14. 10.1016/j.compstruc.2018.04.005 . hal-01996651

\author{
HAL Id: hal-01996651 \\ https://hal.utc.fr/hal-01996651
}

Submitted on 5 Feb 2019

HAL is a multi-disciplinary open access archive for the deposit and dissemination of scientific research documents, whether they are published or not. The documents may come from teaching and research institutions in France or abroad, or from public or private research centers.
L'archive ouverte pluridisciplinaire HAL, est destinée au dépôt et à la diffusion de documents scientifiques de niveau recherche, publiés ou non, émanant des établissements d'enseignement et de recherche français ou étrangers, des laboratoires publics ou privés. 


\title{
Variational formulation of micropolar elasticity using 3D hexahedral finite-element interpolation with incompatible modes
}

\author{
Sara Grbčić ${ }^{\mathrm{a}, \mathrm{b}}$, Adnan Ibrahimbegović ${ }^{\mathrm{b}, *}$, Gordan Jelenić ${ }^{\mathrm{a}}$ \\ ${ }^{a}$ University of Rijeka, Faculty of Civil Engineering, Croatia \\ ${ }^{\mathrm{b}}$ Université de Technologie de Compiègne - Sorbonne Universités, Chair for Computational Mechanics E Insititut Universitaire de France, France
}

\section{A R T I C L E I N F O}

\section{Article history:}

Received 14 February 2018

Accepted 26 April 2018

\section{Keywords:}

Micropolar theory

Finite element method

Incompatible modes

Model validation

\begin{abstract}
A B S T R A C T
A three-dimensional micropolar elasticity is cast in terms of the rigorous variational formulation. The discrete approximation is based on hexahedral finite element using the conventional Lagrange interpolation and enhanced with incompatible modes. The proposed element convergence is checked by performing patch tests which are derived specifically for micropolar finite elements. The element enhanced performance is also demonstrated by modelling two boundary value problems with analytical solutions, both exhibiting the size-effect. The analyzed problems involve a cylindrical plate bending and pure torsion of circular cylinders, which were previously used in the experimental determination of the micropolar material parameters. The numerical results are compared against the analytical solution, and additionally against existing experiments on a polymeric foam for the pure torsion problem. The enhancement due to incompatible modes provides the needed improvement of the element performance in the bending test without negative effects in the pure-torsion test where incompatible modes are not needed. It is concluded that the proposed element is highly suitable for the numerical validation of the experimental procedure.
\end{abstract}

() 2018 Elsevier Ltd. All rights reserved.

\section{Introduction}

Most of the materials are heterogeneous in general, with a specific microstructure that can be represented at a scale particular for the material itself. When this scale is very small, these materials are considered as homogeneous. For such materials (e.g. metals), any microstructure detail is averaged leading to a homogeneous continuum theory. Commonly used is Cauchy's or classical theory that is able to faithfully describe the material behavior. However, when the microstructure scale becomes significantly large compared to the overall scale, assuming the homogenized material, representation based on the classical theory fails. Many newly developed engineering materials increasingly used in engineering, such as fiber-reinforced composites, honeycomb or cellular structured materials or modern polymers belong to the last category. Due to their heterogeneity, such materials exhibit a so-called size-effect phenomenon, which manifests in increased stiffness of smaller specimens made of the same material, which is not recognized in the classical continuum theory. Moreover, in regions of high stress gradients, such as the neighborhoods of

\footnotetext{
* Corresponding author.

E-mail addresses: sara.grbcic@uniri.hr (S. Grbčić), adnan.ibrahimbegovic@utc.fr (A. Ibrahimbegović), gordan.jelenic@uniri.hr (G. Jelenić).
}

holes, notches and cracks, the stress concentration factor as predicted by the classical theory is higher than that observed experimentally. Even more discrepancies between the classical continuum theory and the experimental testing may be observed in dynamics, thermal analysis and fluid mechanics [1]. Due to such anomalies, an alternative continuum model to accurately describe the behavior of such materials is highly needed.

One such model, further discussed in this paper, is the so-called oriented, or Cosserat or micropolar continuum. Namely, different approaches are developed to study the multi-scale nature of the material deformation, by taking into account additional effects consistent with the observed behavior of such heterogeneous materials. One such development accounting for microstructure effects within the limits of continuum mechanics is introducing higher order derivatives or the field gradients, such as the socalled couple-stress or higher-order strain-gradient theories. An alternative approach is introducing additional degrees of freedom, such as micro-stretch or micro-morphic continuum theory [2], to name only a few. Among such theories introducing additional degrees of freedom, we further elaborate upon so-called micropolar continuum theory, usually attributed to the Cosserat brothers [3]. They enriched the Cauchy's theory by adding to the displacement field an independent microrotation field, representing the local rotation of a material point. The detailed exposition of the historical 
development of such theory can be found in [2], who named it the micropolar theory of elasticity. The main goal of this work is to contribute to the further development of such a more general theory, by performing a detailed analysis of some important micropolar boundary value problems.

The ability to include local rotation extends the modeling capabilities, and allows us to take into account the intrinsic material length-scale. However, the additional capabilities come at a cost. In order to describe such a material, even when assumed to be linear elastic, homogeneous and isotropic, it requires six independent material constants, in contrast to only two such constants for the classical continuum. Moreover, the experimental determination of these materials parameters is much more complex, since the experimental verification and their corresponding conceptualisation and interpretation is far from straightforward. The work in [4] is the first attempt to determine all six micropolar material constants by developing experimental and analytical solutions to the boundary value problem, but without particular success in the experimental part since opposite trends between experiments and analytical predictions have been observed. However, by subsequent refinement of Gauthier's and Jahsman's proposed procedure [4], Lakes and his co-workers give the most significant contribution to devising experimental procedures to determine the micropolar material parameters in their analysis of bones [5-7], polymeric foams [8-11] and metal foams [12], based upon measuring the size-effect. As an alternative to the experiments performed by Lakes and his co-workers, the micropolar parameter determination can be based on various homogenization procedures which replaces a larger-scale composite structure, or assembly of particles, by an effective micropolar continuum model. By assuming that a homogeneous Cosserat material is the best approximation of a heterogeneous Cauchy material, the six material parameters of the micropolar continuum may be determined more easily [13-16]. Several recent works of Wheel et al. [17-19] determined the material parameters of highly heterogeneous materials on a larger-scale by comparing the results of experiments and the finite element simulation.

However, the experimental verification of a micropolar material model still remains a great challenge, since a unified procedure to determine the material parameters of micropolar continuum is still lacking. We argue here that the key to understanding and developing more precise experimental procedures lies in the comprehensive numerical analysis of the solution of the corresponding boundary value problem. Such a comprehensive numerical analysis should broaden the range of problems which may be solved and open up new possibilities for the numerical simulation of experimental set-ups. Therefore, the development of the finite elements of high quality is important for the future progress and understanding of the micropolar continuum theory.

An early attempt to model the micropolar constitutive behaviour using the finite-element method is presented in [20] with more authors working on numerical solutions of the micropolar continuum using different finite elements in the linear analysis (e.g. [21-24]). Furthermore, in addition to the standard finiteelement procedures, non-standard finite-element methods, such as the control-volume-based finite-element method [25,17] have been used to model micropolar finite elements.

The objective of this paper is to present one high quality element for 3D simulations. More precisely, we propose a highperformance three-dimensional micropolar hexahedral finite element, using conventional Lagrange interpolation enhanced with the so-called incompatible modes [26,27]. The proposed element performance is tested against the analytical boundary value problems derived by Gauthier and Jahsman [4] and experiments per- formed by Lakes and co-workers [5-12]. In the framework of the classical elasticity the incompatible displacement modes are first added to the isoparametric elements (e.g. see [26-29]). The main benefit of incompatible modes in the classical continuum framework is to avoid shear locking, as shown already in early 1970 s [30]. In bending of isoparametric 4-node 2D or 8-node 3D finite elements, the absence of quadratic polynomials in the displacement field approximation predicts the shear strain in pure bending incorrectly. This is called the shear-locking effect [31]. Even with higher-order elements producing better results in pure-bending tests, the maximum possible reduction of computational cost is always a worthwhile goal. The proposed solution is to enrich the displacement interpolation of the corresponding element with quadratic displacement interpolation modes, requiring internal element degrees of freedom and leading to incompatibility of the displacement field. When first introduced into 2D quadrilateral isoparametric finite elements [30], the method was received with skepticism in the finite element method research community, since the displacement compatibility between finite elements was at that time considered to be absolutely mandatory [32]. The use of the incompatible-mode method for low-order elements in both two- and three-dimensional problems is nowadays common, leading to the most impressive performance not only in bending, but also elsewhere, e.g. when modelling cracking $[33,29]$ and two-phase materials [34]. A detailed exposition of 1D, 2D and 3D finite elements with incompatible modes in classical elasticity is presented in [35].

In the framework of micropolar elasticity, the idea of enhancing the displacement field of standard finite element is already recognised in [36], where authors analyzed straight and curved beam problems subject to shear loading. Only 2D problems have been analyzed in [36] and the numerical results have not always converged to the reference analytical solution. In the present work, the high performance of the presented finite element is demonstrated by successful analysis of both 2D and 3D problems. Moreover, our ability to deliver the solution that can converge to reference values was confirmed for both bending and torsion.

\section{Micropolar continuum model formulation}

The fundamental relations of linear micropolar elasticity applied to a homogeneous and isotropic material are outlined in this section. We consider a continuous body $\mathcal{B}$, of volume $V$ and boundary surface $S$ in the deformed state under the influence of external actions consisting of distributed body force $\mathbf{p}_{\mathrm{v}}$ and body moment $\mathbf{m}_{\mathrm{v}}$ and distributed surface force $\mathbf{p}_{\mathrm{s}}$ and surface moment $\mathbf{m}_{\text {s }}$. By generalising the Cauchy stress principle (see [37]), at an internal material point $X$, with the position vector $\mathbf{x}$, with respect to a chosen spatial frame of reference at time $t$, we prove the existence of a second-order Cauchy stress tensor $\boldsymbol{\sigma}(\mathbf{x}, t)$ and an additional second-order couple-stress tensor $\boldsymbol{\mu}(\mathbf{x}, t)$.

\subsection{Equilibrium equations}

By analysing the static equilibrium of a differential volume $d V$ in the deformed state, we can obtain the force equilibrium equation

$\boldsymbol{\sigma} \nabla+\mathbf{p}_{\mathrm{v}}=\mathbf{0}$,

where $\nabla$ is the differential operator nabla (e.g. see [29]), and the moment equilibrium equation

$\boldsymbol{\mu} \nabla+\mathbf{a}+\mathbf{m}_{\mathrm{v}}=\mathbf{0}$. 
In (2) above, $\mathbf{a}$ is twice the axial vector of the skew-symmetric part of the stress tensor $\boldsymbol{\sigma}_{\mathrm{a}}=\frac{1}{2}\left(\boldsymbol{\sigma}-\boldsymbol{\sigma}^{\mathrm{T}}\right)$, i.e.

$$
\mathbf{a}=2 \operatorname{axial}\left(\boldsymbol{\sigma}_{\mathrm{a}}\right)=\left\{\begin{array}{lll}
\sigma_{32}-\sigma_{23} & -\sigma_{31}+\sigma_{13} & \sigma_{21}-\sigma_{12}
\end{array}\right\}^{\mathrm{T}}, \quad 2 \boldsymbol{\sigma}_{\mathrm{a}}=\widehat{\mathbf{a}},
$$

where a superimposed hat on a vector field $(\cdot)$ denotes a skew-symmetric cross-product operator such that $\widehat{(\cdot)} \mathbf{v}=(\cdot) \times \mathbf{v}$ for any 3D vector $\mathbf{v}$. Equilibrium equations written using the Einstein summation convention on repeated indices are thus equal to

$\sigma_{i j . j}+p_{v i}=0, \quad \mu_{i j, j}-\varepsilon_{i j k} \sigma_{j k}+m_{v i}=0$,

where the first index denotes the direction of the stress or axis of the couple stress component with respect to the coordinate base and the second index denotes the direction of the surface normal. The comma denotes differentiation with respect to spatial coordinate and $\varepsilon_{i j k}$ denotes the permutation tensor (Levi-Civita tensor).

By analyzing the differential surface $d S$ subject to surface loading, the following natural boundary conditions are obtained:

$$
\boldsymbol{\sigma} \mathbf{n}=\mathbf{p}_{\mathrm{s}} \Longleftrightarrow \sigma_{i j} n_{j}=p_{s i}, \quad \boldsymbol{\mu} \mathbf{n}=\mathbf{m}_{\mathrm{s}} \quad \Longleftrightarrow \quad \mu_{i j} n_{j}=m_{s i},
$$

where $\mathbf{n}$ is the outward unit normal to the surface.

\subsection{Kinematic equations}

In relation to the classical continuum theory, in the micropolar continuum theory we have a displacement field $\mathbf{u}(\mathbf{x})$ and an additional microrotation field $\boldsymbol{\varphi}(\mathbf{x})$, representing the local rotation of the point $X$ which is completely independent of the displacement field. Consequently, the microrotation $\varphi$ is also independent from the rotation part of the displacement gradient, i.e. from the macrorotation $\omega$ of the classical continuum theory (see [37]). The micropolar strain tensor $\boldsymbol{\epsilon}$ is defined as

$\boldsymbol{\epsilon}=\operatorname{grad} \mathbf{u}-\widehat{\boldsymbol{\varphi}}=\mathbf{u} \otimes \nabla-\widehat{\boldsymbol{\varphi}} \quad \Longleftrightarrow \quad \epsilon_{i j}=u_{i, j}+\varepsilon_{i j k} \varphi_{k}$,

The normal strains in the micropolar continuum theory $\epsilon_{11}, \epsilon_{22}, \epsilon_{33}$ are equal to those in the classical continuum theory, which means that the microrotation $\varphi$ does not contribute to stretching or shortening of the generic fibre. The influence of the microrotation is present only in shear strains $\epsilon_{i j}, i, j=1,2,3, i \neq j$, which are defined to be equal to the difference between the change of inclination of a generic fibre during deformation and the microrotation $\varphi$. The independent rotation field $\varphi$ also gives rise to a corresponding micropolar curvature tensor

$\boldsymbol{\kappa}=\operatorname{grad} \boldsymbol{\varphi}=\boldsymbol{\varphi} \otimes \nabla \quad \Longleftrightarrow \quad \kappa_{i j}=\varphi_{i, j}$,

where the diagonal terms represent torsional strains. We note that the so-called couple-stress theory (see [38]) is a special case of the micropolar continuum theory where the microrotation vector $\varphi$ is equal to the macrorotation vector $\omega$. Thus, in the couple-stress theory, the curvature tensor involves second derivatives of the displacement field. When these derivatives are neglected, the curvature tensor also vanishes and the couple-stress theory reduces to the classical continuum theory.

\subsection{Constitutive equations}

In a homogeneous isotropic linear elastic micropolar continuum, the second-order stress and strain tensors $\boldsymbol{\sigma}$ and $\boldsymbol{\epsilon}$ are related via a constant isotropic fourth-order constitutive tensor $\mathbf{T}$ such that in the component form we have [39]

$$
\begin{aligned}
& \sigma_{i j}=T_{i j p q} \epsilon_{p q}, \\
& T_{i j p q}=\lambda \delta_{i j} \delta_{p q}+\mu\left(\delta_{i p} \delta_{j q}+\delta_{i q} \delta_{j p}\right)+v\left(\delta_{i p} \delta_{j q}-\delta_{i q} \delta_{j p}\right),
\end{aligned}
$$

where $\lambda$ and $\mu$ are the Lamé constants, $v$ is another material constant and $\delta_{i j}$ is the Kronecker symbol. The couple-stress tensor $\boldsymbol{\mu}$ is related to the curvature tensor $\boldsymbol{\kappa}$ in a completely analogous way, i.e.

$\sigma_{i j}=\lambda \epsilon_{p p} \delta_{i j}+(\mu+v) \epsilon_{i j}+(\mu-v) \epsilon_{j i}$,

$\mu_{i j}=\alpha \kappa_{p p} \delta_{i j}+(\beta+\gamma) \kappa_{i j}+(\beta-\gamma) \kappa_{j i}$

where $\alpha, \beta, \gamma$ are three additional material parameters. The following restrictions on the material parameters should hold in order to enforce positive definiteness of the constitutive tensors: $3 \lambda+2 \mu>0, \mu>0, v>0,3 \alpha+2 \beta>0, \beta>0$ and $\gamma>0$. Note that all the stress and strain tensors are in general non-symmetric.

These material parameters are related to a set of engineering (measurable) parameters, via [40]:

$$
\begin{aligned}
& \lambda=\frac{2 n G}{1-2 n}, \quad \mu=G, \quad v=\frac{G N^{2}}{1-N^{2}}, \\
& \alpha=\frac{2 G l_{t}^{2}(1-\psi)}{\psi}, \quad \beta=G l_{t}^{2}, \quad \gamma=G\left(4 l_{b}^{2}-l_{t}^{2}\right) .
\end{aligned}
$$

Parameter $G$ represents the shear modulus, $n$ is Poisson's ratio, $l_{t}$ the characteristic length for torsion and $l_{b}$ the characteristic length for bending. Characteristic length variables quantify the influence of the microstructure on the macro-behavior of the material and have the dimension of length. Their values are of an order of magnitude of material particle-, grain- or cell-size, depending on the material microstructure. Parameter $N$ represents the coupling number that is a dimensionless measure of the degree of coupling between the microrotation vector $\varphi$ and the macrorotation vector $\omega$, with the restricted value $N \in\langle 0,1\rangle$. Consequently, $v$ quantifies the degree of coupling between macro- and microrotation effects. When $N$ tends to the limit $N=1$, parameter $v$ tends to infinity, which is the case of the so-called couple-stress elasticity [41]. Finally, parameter $\psi \in\left\langle 0, \frac{3}{2}\right\rangle$ represents the dimensionless polar ratio of rotation sensitivity (a quantity which relates the torsional strains in a way analogous to that in which Poisson's ratio relates the normal strains).

\section{Weak form of the boundary value problem in 3D micropolar elasticity}

For constructing a numerical solution procedure of the boundary value problem, we abandon its strong (or differential) form in favor of the corresponding weak (or integral) form. The displacement-type weak formulation is obtained by means of the principle of virtual work stating that the difference between virtual works of external and internal forces should vanish, i.e.

$G(\mathbf{u}, \boldsymbol{\varphi} ; \overline{\mathbf{u}}, \overline{\boldsymbol{\varphi}})=G^{\text {int }}(\mathbf{u}, \boldsymbol{\varphi} ; \overline{\mathbf{u}}, \overline{\boldsymbol{\varphi}})-G^{\text {ext }}(\overline{\mathbf{u}}, \overline{\boldsymbol{\varphi}})=0$.

The virtual work of internal and external forces can be expressed as

$$
\begin{aligned}
& G^{i n t}(\mathbf{u}, \boldsymbol{\varphi} ; \overline{\mathbf{u}}, \overline{\boldsymbol{\varphi}})=\int_{V}(\overline{\boldsymbol{\epsilon}}: \boldsymbol{\sigma}+\overline{\boldsymbol{\kappa}}: \boldsymbol{\mu}) d V \\
& G^{\text {ext }}(\overline{\mathbf{u}}, \overline{\boldsymbol{\varphi}})=\int_{V}\left(\overline{\mathbf{u}} \cdot \mathbf{p}_{v}+\overline{\boldsymbol{\varphi}} \cdot \mathbf{m}_{v}\right) d V+\int_{S}\left(\overline{\mathbf{u}} \cdot \mathbf{p}_{s}+\overline{\boldsymbol{\varphi}} \cdot \mathbf{m}_{s}\right) d S,
\end{aligned}
$$

where $\overline{\mathbf{u}}$ and $\bar{\varphi}$ are the virtual displacements and virtual microrotation vectors and $\overline{\boldsymbol{\epsilon}}$ and $\overline{\boldsymbol{\kappa}}$ are the corresponding tensors of virtual micropolar strains and curvatures, respectively. In order to obtain the numerical solution of the problem, the kinematic fields have to be approximated using chosen interpolations. In general, the real and virtual kinematic fields interpolation are chosen the same leading to $\mathbf{u}^{h}=\mathbf{N}_{\mathrm{u}} \mathbf{d}^{e}, \varphi^{h}=\mathbf{N}_{\varphi} \mathbf{d}^{e}, \overline{\mathbf{u}}^{h}=\mathbf{N}_{\mathrm{u}} \overline{\mathbf{d}}^{e}, \bar{\varphi}^{h}=\mathbf{N}_{\varphi} \overline{\mathbf{d}}^{e}$. More precisely, $\mathbf{N}_{\mathrm{u}}$ and $\mathbf{N}_{\varphi}$ represent the matrices of interpolation functions for the displacement and microrotation field, and $\mathbf{d}^{e}$ and $\overline{\mathbf{d}}^{e}$ represent 
the real and virtual vector of element nodal degrees of freedom, respectively. Superscript $h$ denotes the finite-dimensional approximation and $e$ the element level. After introducing the chosen interpolation of the kinematic fields and their virtual counterparts into (11) we obtain the interpolated element internal and external virtual works as

$G^{\text {int }, e}\left(\mathbf{d}^{e} ; \overline{\mathbf{d}}^{e}\right)=\overline{\mathbf{d}}^{e \mathrm{~T}} \mathbf{K}^{e} \mathbf{d}^{e}, \quad G^{e x t, e}\left(\overline{\mathbf{d}}^{e}\right)=\overline{\mathbf{d}}^{e \mathrm{~T}} \mathbf{f}^{e}$,

where $\mathbf{K}^{e}$ and $\mathbf{f}^{e}$ represent the element stiffness matrix and external force vector. The global internal and external virtual works are obtained by assembly over $n_{\text {elem }}$ as the total number of elements in the mesh, with $\mathbb{A}$ as the finite-element assembly operator [29] as

$G^{\text {int }}(\mathbf{d} ; \overline{\mathbf{d}})=\underset{e=1}{n_{\text {elem }}} G^{\text {int }, e}\left(\mathbf{d}^{e} ; \overline{\mathbf{d}}^{e}\right) \equiv \overline{\mathbf{d}}^{\mathrm{T}} \mathbf{K d}$,

$G^{e x t}(\overline{\mathbf{d}})=\underset{e=1}{n_{\text {elem }}} G^{\text {ext,e }}\left(\overline{\mathbf{d}}^{e}\right) \equiv \overline{\mathbf{d}}^{\mathrm{T}} \mathbf{f}$,

with $\mathbf{d}$ and $\overline{\mathbf{d}}$ being the global vectors of real and virtual displace-

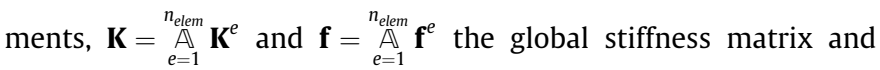
external force vector. For arbitrary values of virtual parameters, $\forall \overline{\mathbf{d}}$, the approximated principle of virtual work leads to the basic algebraic equations of the finite element method $\mathbf{K d}=\mathbf{f}$.

\section{Lagrangian elements with incompatible modes interpolation}

In this work two different interpolations are tested. Both interpolations are applied on an isoparametric trilinear hexahedral finite element with eight nodes and six degrees of freedom per node (three displacements $u_{x}, u_{y}, u_{z}$ and three microrotations $\varphi_{x}, \varphi_{y}, \varphi_{z}$ ) with the numbering convention as shown in Fig. 1. The first type is the conventional trilinear Lagrange interpolation defined in the natural coordinate system, chosen for both displacement and microrotation fields, and the corresponding finite element is called Hex8. The second interpolation consists of the Lagrange interpolations for displacement and microrotation fields, but with the displacement interpolation additionally enriched by incompatible modes. The derived finite element is referred to as Hex8IM.

For the element Hex8IM the real and virtual displacement field interpolations are defined as

$$
\begin{aligned}
\mathbf{u}^{h} & =\sum_{i=1}^{8} N_{i}(\xi, \eta, \zeta) \mathbf{u}_{i}^{e}+\sum_{i=1}^{3} M_{i}(\xi, \eta, \zeta) \boldsymbol{\alpha}_{i}^{e}=\mathbf{N}_{u} \mathbf{d}^{e}+\mathbf{N}_{e n h} \boldsymbol{\alpha}^{e}, \\
\overline{\mathbf{u}}^{h} & =\sum_{i=1}^{8} N_{i}(\xi, \eta, \zeta) \overline{\mathbf{u}}_{i}^{e}+\sum_{i=1}^{3} M_{i}(\xi, \eta, \zeta) \overline{\boldsymbol{\alpha}}_{i}^{e}=\mathbf{N}_{u} \overline{\mathbf{d}}^{e}+\mathbf{N}_{e n h} \overline{\boldsymbol{\alpha}}^{e},
\end{aligned}
$$

where
$N_{i}(\xi, \eta, \zeta)=\frac{1}{8}\left(1+\xi_{a} \xi\right)\left(1+\eta_{a} \eta\right)\left(1+\zeta_{a} \zeta\right)$
$\xi_{a}= \pm 1, \eta_{a}= \pm 1, \zeta_{a}= \pm 1, i=1, \ldots, 8$

represent the Lagrange trilinear isoparametric shape functions [31], $\mathbf{u}_{i}^{e}=\left\langle u_{x i} u_{y i} u_{z i}\right\rangle^{\mathrm{T}}$ is the vector of element nodal displacements at node $i$, and $\alpha_{i}^{e}=\left\langle\alpha_{1 i} \alpha_{2 i} \alpha_{3 i}\right\rangle^{\mathrm{T}}$ is the vector of the element parameters for the incompatible shape functions chosen as: $M_{1}=1-\xi^{2}, M_{2}=1-\eta^{2}$, and $M_{3}=1-\zeta^{2}$. From (15) we can see that the displacement field interpolation consists of the conventional (compatible) part $\mathbf{N}_{u} \mathbf{d}^{e}$ and the enhanced (incompatible) part $\mathbf{N}_{e n h} \boldsymbol{\alpha}^{e}$. In the compatible part, defining the complete displacement field interpolation of the Hex8 element, the vector of element nodal degrees of freedom is defined as $\mathbf{d}^{e}=\left\langle\mathbf{d}_{1}^{e} \mathbf{d}_{2}^{e} \cdots \mathbf{d}_{8}^{e}\right\rangle$, where $\mathbf{d}_{i}^{e}=\left\langle u_{x i} u_{y i} u_{z i} \varphi_{x i} \varphi_{y i} \varphi_{z i}\right\rangle^{\mathrm{T}}, i$ being the node number, and the matrix of Lagrange interpolation functions is defined as $\mathbf{N}_{u}=\left[\mathbf{N}_{1} \mathbf{0} \cdots \mathbf{N}_{8} \mathbf{0}\right]$, with explicit form of the sub-matrix of Lagrange interpolation functions as

$\mathbf{N}_{i}=\left[\begin{array}{ccc}N_{i} & 0 & 0 \\ 0 & N_{i} & 0 \\ 0 & 0 & N_{i}\end{array}\right]$

and $\mathbf{0}$ as a $3 \times 3$ zero-matrix. Similarly, in the enhanced part with $\boldsymbol{\alpha}^{e}=\left\langle\boldsymbol{\alpha}_{1}^{e} \boldsymbol{\alpha}_{2}^{e} \boldsymbol{\alpha}_{3}^{e}\right\rangle^{\mathrm{T}}$ as the element vector of additional degrees of freedom, the matrix of incompatible shape functions is written as

$\mathbf{N}_{e n h}=\left[\begin{array}{ccccccccc}M_{1} & 0 & 0 & M_{2} & 0 & 0 & M_{3} & 0 & 0 \\ 0 & M_{1} & 0 & 0 & M_{2} & 0 & 0 & M_{3} & 0 \\ 0 & 0 & M_{1} & 0 & 0 & M_{2} & 0 & 0 & M_{3}\end{array}\right]$.

The virtual fields $\overline{\mathbf{u}}_{i}^{e}, \overline{\boldsymbol{\alpha}}_{i}^{e}, \overline{\mathbf{d}}^{e}$ and $\overline{\boldsymbol{\alpha}}^{e}$ are defined analogously.

The real and virtual microrotation fields for both Hex8 and Hex8IM are interpolated by using only the standard Lagrange interpolation:

$\boldsymbol{\varphi}^{h}=\sum_{i=1}^{8} N_{i}(\xi, \eta, \zeta) \boldsymbol{\varphi}_{i}^{e}=\mathbf{N}_{\varphi} \mathbf{d}^{e}, \quad \overline{\boldsymbol{\varphi}}^{h}=\sum_{i=1}^{8} N_{i}(\xi, \eta, \zeta) \overline{\boldsymbol{\varphi}}_{i}^{e}=\mathbf{N}_{\varphi} \overline{\mathbf{d}}^{e}$,

where $\boldsymbol{\varphi}_{i}^{e}=\left\langle\varphi_{x i} \varphi_{y i} \varphi_{z i}\right\rangle^{\mathrm{T}}$ is the vector of nodal microrotations at node $i$ and $\mathbf{N}_{\varphi}=\left[\mathbf{O} \mathbf{N}_{1} \ldots \mathbf{O} \mathbf{N}_{8}\right]$.

It is important to note that the reference configuration of the isoparametric element is still defined only with the compatible shape functions, i.e. the mapping between the natural coordinate system and the global coordinate system is defined as $\mathbf{x}^{h}=\sum_{i=1}^{8} N_{i}(\xi, \eta, \zeta) \mathbf{x}_{i}^{e}$ where $\mathbf{x}_{i}^{e}=\left\langle x_{i} y_{i} z_{i}\right\rangle^{\mathrm{T}}$ represents the vector of element nodal coordinates at node $i$.

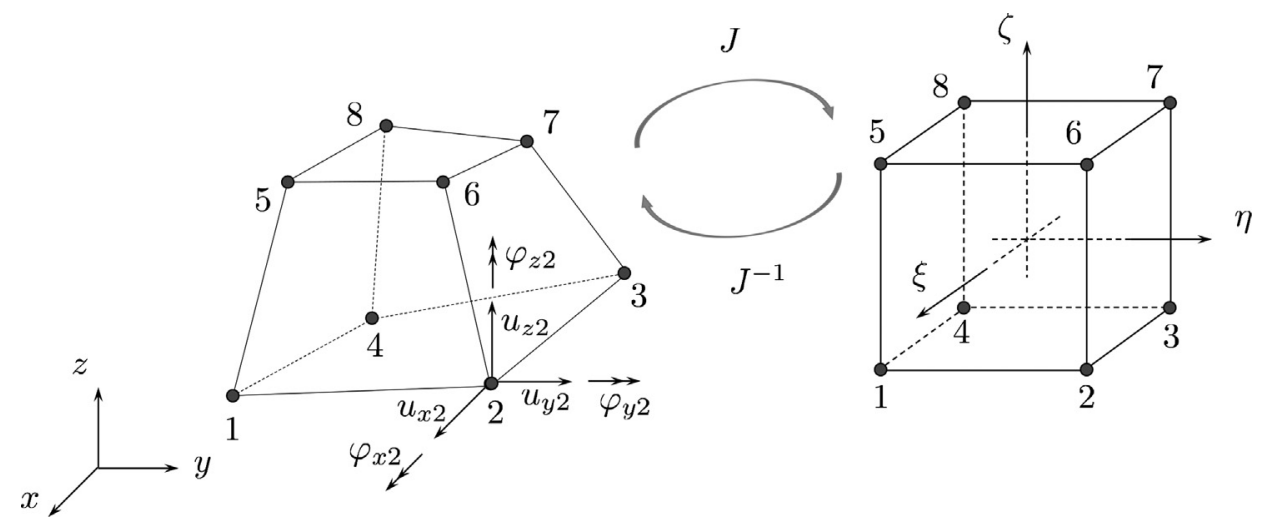

Fig. 1. Hexahedral finite element with eight nodes. 
By introducing the interpolation into the kinematic equations we obtain the vector of interpolated micropolar strain field

$$
\begin{aligned}
\boldsymbol{\epsilon}^{h} & =\left\langle\epsilon_{11} \epsilon_{12} \epsilon_{13} \epsilon_{21} \epsilon_{22} \epsilon_{23} \epsilon_{31} \epsilon_{32} \epsilon_{33}\right\rangle^{\mathrm{T}} \\
& =\sum_{i=1}^{8} \mathbf{B}_{u_{i}} \mathbf{d}_{i}^{e}+\sum_{i=1}^{8} \mathbf{Q}_{\varphi_{i}} \mathbf{d}_{i}^{e}+\sum_{i=1}^{3} \mathbf{G}_{i} \boldsymbol{\alpha}_{i}^{e},
\end{aligned}
$$

and the vector of interpolated curvature field

$\boldsymbol{\kappa}^{h}=\left\langle\begin{array}{lllllllll}\kappa_{11} & \kappa_{12} & \kappa_{13} & \kappa_{21} & \kappa_{22} & \kappa_{23} & \kappa_{31} & \kappa_{32} & \kappa_{33}\end{array}\right\rangle^{\mathrm{T}}=\sum_{i=1}^{8} \mathbf{B}_{\varphi_{i}} \mathbf{d}_{i}^{e}$,

where matrices $\mathbf{B}_{u_{i}}=\left[\mathbf{B}_{i} \mathbf{0}\right]$ and $\mathbf{B}_{\varphi_{i}}=\left[\begin{array}{l}\mathbf{0} \\ \mathbf{B}_{i}\end{array}\right]$ represent the matrices of global derivatives of the compatible shape functions, matrix $\mathbf{Q}_{\varphi i}=\left[\mathbf{0} \mathbf{Q}_{i}\right]$ is the matrix of compatible shape functions defining the presence of microrotations in the definition of micropolar strains, matrix $\mathbf{G}_{i}$ is the matrix of global derivatives of incompatible shape functions and $\mathbf{0}$ is a $9 \times 3$ zero matrix, where the submatrices are defined as

$\mathbf{B}_{i}=\left[\begin{array}{ccc}\frac{\partial N_{i}}{\partial x} & 0 & 0 \\ \frac{\partial N_{i}}{\partial y} & 0 & 0 \\ \frac{\partial N_{i}}{\partial z} & 0 & 0 \\ 0 & \frac{\partial N_{i}}{\partial x} & 0 \\ 0 & \frac{\partial N_{i}}{\partial y} & 0 \\ 0 & \frac{\partial N_{i}}{\partial z} & 0 \\ 0 & 0 & \frac{\partial N_{i}}{\partial x} \\ 0 & 0 & \frac{\partial N_{i}}{\partial y} \\ 0 & 0 & \frac{\partial N_{i}}{\partial z}\end{array}\right], \mathbf{Q}_{i}=\left[\begin{array}{ccc}0 & 0 & 0 \\ 0 & 0 & N_{i} \\ 0 & -N_{i} & 0 \\ 0 & 0 & -N_{i} \\ 0 & 0 & 0 \\ N_{i} & 0 & 0 \\ 0 & N_{i} & 0 \\ -N_{i} & 0 & 0 \\ 0 & 0 & 0\end{array}\right], \mathbf{G}_{i}=\left[\begin{array}{ccc}\frac{\partial M_{i}}{\partial x} & 0 & 0 \\ \frac{\partial M_{i}}{\partial y} & 0 & 0 \\ \frac{\partial M_{i}}{\partial z} & 0 & 0 \\ 0 & \frac{\partial M_{i}}{\partial x} & 0 \\ 0 & \frac{\partial M_{i}}{\partial y} & 0 \\ 0 & \frac{\partial M_{i}}{\partial z} & 0 \\ 0 & 0 & \frac{\partial M_{i}}{\partial x} \\ 0 & 0 & \frac{\partial M_{i}}{\partial y} \\ 0 & 0 & \frac{\partial M_{i}}{\partial z}\end{array}\right]$.

The global derivatives of compatible and incompatible shape functions are given by the usual chain rule expressions using the Jacobian matrix $\mathbf{J}=\frac{\partial(x, y, z)}{\partial(\xi, \eta, \zeta)}[31]$, i.e.

$$
\left\{\begin{array}{c}
\frac{\partial N_{i}}{\partial x} \\
\frac{\partial N_{i}}{\partial y} \\
\frac{\partial N_{i}}{\partial z}
\end{array}\right\}=\underbrace{\left[\begin{array}{lll}
\frac{\partial \xi}{\partial x} & \frac{\partial \eta}{\partial x} & \frac{\partial \zeta}{\partial x} \\
\frac{\partial \xi}{\partial y} & \frac{\partial \eta}{\partial y} & \frac{\partial \zeta}{\partial y} \\
\frac{\partial \xi}{\partial z} & \frac{\partial \eta}{\partial z} & \frac{\partial \zeta}{\partial z}
\end{array}\right]}_{\mathbf{J}^{-1}}\left\{\begin{array}{c}
\frac{\partial N_{i}}{\partial \xi} \\
\frac{\partial N_{i}}{\partial \eta} \\
\frac{\partial N_{i}}{\partial \zeta}
\end{array}\right\},\left\{\begin{array}{c}
\frac{\partial M_{i}}{\partial x} \\
\frac{\partial M_{i}}{\partial y} \\
\frac{\partial M_{i}}{\partial z}
\end{array}\right\}=\underbrace{\left[\begin{array}{ccc}
\frac{\partial \xi}{\partial x} & \frac{\partial \eta}{\partial x} & \frac{\partial \zeta}{\partial x} \\
\frac{\partial \xi}{\partial y} & \frac{\partial \eta}{\partial y} & \frac{\partial \zeta}{\partial y} \\
\frac{\partial \xi}{\partial z} & \frac{\partial \eta}{\partial z} & \frac{\partial \zeta}{\partial z}
\end{array}\right]}_{\mathbf{J}^{-1}}\left\{\begin{array}{c}
\frac{\partial M_{i}}{\partial \xi} \\
\frac{\partial M_{i}}{\partial \eta} \\
\frac{\partial M_{i}}{\partial \zeta}
\end{array}\right\} .
$$

Before proceeding to the derivation of the element stiffness matrix, we have to take into account the finite element convergence criteria which states that any enhancement beyond the standard definition of the strain field has to vanish for a state of constant strain. In other words, any enhanced strain field must satisfy the stress orthogonality condition [29]. When enhancing the conventional interpolation functions, the condition which has to be satisfied when performing a patch test of order $n$, is that all the enhancement of order $(n+1)$ has to vanish. By imposing the requirement that the strain energy associated with the incompatible modes under the state of constant stress has to vanish we obtain the following equation [26]:

$\frac{1}{2} \boldsymbol{\sigma}^{\mathrm{T}} \int_{V^{e}} \mathbf{G}_{i} d V \boldsymbol{\alpha}^{e}=0 \Rightarrow \int_{V^{e}} \mathbf{G}_{i} d V=0$,

where $V^{e}$ is the element volume and $\boldsymbol{\sigma}=\left\langle\sigma_{11} \sigma_{12} \sigma_{13} \sigma_{21} \sigma_{22} \sigma_{23}\right.$ $\left.\sigma_{31} \sigma_{32} \sigma_{33}\right\rangle^{\mathrm{T}}$ is the element stress vector. This can be satisfied by adding a constant correction matrix $\mathbf{G}_{\mathbf{c} i}$ to the matrix $\mathbf{G}_{i}$, i.e. $\tilde{\mathbf{G}}_{i}=\mathbf{G}_{i}+\mathbf{G}_{\mathbf{c} i}$ such that
$\int_{V^{e}} \tilde{\mathbf{G}}_{i} d V=\int_{V^{e}}\left(\mathbf{G}_{\mathbf{c} i}+\mathbf{G}_{i}\right) d V=0$,

which, by the fact that $\mathbf{G}_{\mathbf{c} i}$ is constant, leads to the following modification of matrix $\mathbf{G}_{i}$ [26]:

$\tilde{\mathbf{G}}_{i}=\mathbf{G}_{i}-\frac{1}{V^{e}} \int_{V^{e}} \mathbf{G}_{i} d V$.

By introducing the interpolation of the kinematic fields into the weak formulation we obtain a system of two equations defined at the element level, i.e.

$$
\left\langle\overline{\mathbf{d}}^{\mathrm{e}^{\mathrm{T}}} \overline{\boldsymbol{\alpha}}^{\mathrm{e}^{\mathrm{T}}}\right\rangle\left(\left[\begin{array}{cc}
\mathbf{K}^{e} & \mathbf{F}^{\mathrm{T}} \\
\mathbf{F}^{e} & \mathbf{H}^{e}
\end{array}\right]\left\{\begin{array}{l}
\mathbf{d}^{e} \\
\boldsymbol{\alpha}^{e}
\end{array}\right\}\right)=\left\{\begin{array}{l}
\mathbf{f}^{e} \\
\mathbf{0}
\end{array}\right\},
$$

where the obtained matrices are equal to

$$
\begin{aligned}
\mathbf{K}^{e} & =\int_{V^{e}}\left(\left(\mathbf{B}_{\mathbf{u}}^{\mathrm{T}}+\mathbf{Q}_{\varphi}^{\mathrm{T}}\right) \mathbf{C}_{\mathbf{1}}\left(\mathbf{B}_{\mathbf{u}}+\mathbf{Q}_{\varphi}\right)+\mathbf{B}_{\varphi}^{\mathrm{T}} \mathbf{C}_{\mathbf{2}} \mathbf{B}_{\varphi}\right) d V, \\
\mathbf{F}^{e} & =\int_{V^{e}} \tilde{\mathbf{G}}^{\mathrm{T}} \mathbf{C}_{\mathbf{1}}\left(\mathbf{B}_{\mathbf{u}}+\mathbf{Q}_{\varphi}\right) d V, \\
\mathbf{H}^{e} & =\int_{V^{e}} \tilde{\mathbf{G}}^{\mathrm{T}} \mathbf{C}_{\mathbf{1}} \tilde{\mathbf{G}} d V,
\end{aligned}
$$

where $\quad \mathbf{B}_{\mathbf{u}}=\left[\begin{array}{llll}\mathbf{B}_{u_{1}} & \mathbf{B}_{u_{2}} \ldots \mathbf{B}_{u_{8}}\end{array}\right]^{\mathrm{T}}, \quad \mathbf{Q}_{\varphi}=\left[\begin{array}{lll}\mathbf{Q}_{\varphi_{1}} & \mathbf{Q}_{\varphi_{2}} \ldots \mathbf{Q}_{\varphi_{8}}\end{array}\right], \quad \mathbf{B}_{\varphi}=\left[\begin{array}{l}\mathbf{B}_{\varphi_{1}} \\ \text { wat }\end{array}\right.$ $\left.\mathbf{B}_{\varphi_{2}} \ldots \mathbf{B}_{\varphi_{8}}\right]^{\mathrm{T}}, \tilde{\mathbf{G}}=\left[\tilde{\mathbf{G}}_{1} \tilde{\mathbf{G}}_{2} \tilde{\mathbf{G}}_{3}\right]^{\mathrm{T}}$, and $\mathbf{C}_{\mathbf{1}}$ and $\mathbf{C}_{\mathbf{2}}$ are $9 \times 9$ constitutive matrices defined as

$$
\mathbf{C}_{\mathbf{1}}=\left[\begin{array}{ccccccccc}
(\lambda+2 \mu) & 0 & 0 & 0 & \lambda & 0 & 0 & 0 & \lambda \\
0 & (\mu+v) & 0 & (\mu-v) & 0 & 0 & 0 & 0 & 0 \\
0 & 0 & (\mu+v) & 0 & 0 & 0 & (\mu-v) & 0 & 0 \\
0 & (\mu-v) & 0 & (\mu+v) & 0 & 0 & 0 & 0 & 0 \\
\lambda & 0 & 0 & 0 & (\lambda+2 \mu) & 0 & 0 & 0 & \lambda \\
0 & 0 & 0 & 0 & 0 & (\mu+v) & 0 & (\mu-v) & 0 \\
0 & 0 & (\mu-v) & 0 & 0 & 0 & (\mu+v) & 0 & 0 \\
0 & 0 & 0 & 0 & 0 & (\mu-v) & 0 & (\mu+v) & 0 \\
\lambda & 0 & 0 & 0 & \lambda & 0 & 0 & 0 & (\lambda+2 \mu)
\end{array}\right]
$$

with a corresponding result for $\mathbf{C}_{2}$ in which $\alpha, \beta, \gamma$ replace $\lambda, \mu, v$. In order to eliminate the presence of unknown incompatible-mode parameters $\boldsymbol{\alpha}^{e}$, we have to perform the so-called static condensation [28]. The static condensation is accomplished by first expressing from the second equation $\boldsymbol{\alpha}^{e}=-\mathbf{H}^{e^{-1}} \mathbf{F}^{e} \mathbf{d}^{e}$ and then introducing it into the first equation. Consequently, we obtain the reduced form of the element stiffness matrix

$$
\widetilde{\mathbf{K}}^{e}=\mathbf{K}^{e}-\mathbf{F}^{\mathbf{T}^{\mathrm{T}}} \mathbf{H}^{e^{-1}} \mathbf{F}^{e}
$$

From this point on, we can proceed towards the standard finite element assembly accounting for all element contributions, i.e.

$\mathbf{K d}=\mathbf{f} \Rightarrow \mathbf{d} ; \quad \mathbf{K}=\underset{e=1}{n_{\text {elem }}} \tilde{\mathbf{K}}^{e} ; \quad \mathbf{f}=\underset{e=1}{n_{\text {elem }}} \mathbf{f}^{e}$.

Having the nodal displacement values obtained, we can recover the corresponding element displacements $\mathbf{d}^{e}$ through the connectivity matrix $\mathbf{d}=\mathbf{L}^{e} \mathbf{d}^{e}$ which allows to obtain the incompatible mode parameters $\boldsymbol{\alpha}^{e}=-\mathbf{H}^{e^{-1}} \mathbf{F}^{e} \mathbf{d}^{e}$ and recover the micropolar strains $\boldsymbol{\epsilon}^{h}$ in (20). Stresses $\boldsymbol{\sigma}^{h}$ in Gauss points are then obtained from the constitutive equations. The curvatures $\boldsymbol{\kappa}^{h}$ are obtained in a conventional manner, directly from the element displacements, as shown in Eq. (21) and again, using the constitutive equation, we obtain the couple-stresses in Gauss points $\boldsymbol{\mu}^{h}$. 


\section{Numerical examples}

In this section the performance of the conventional eight-node hexahedral micropolar finite element Hex8 and the enhanced element with incompatible modes Hex8IM is tested in several numerical examples. Both finite elements are coded within the Finite Element Analysis Program (FEAP) [42]. In the first example (Section 5.1), the finite-element verification is performed through the so-called patch test [31] on a regular mesh, which represents a standard method for testing the finite element convergence. In the second example (Section 5.2) a set of displacement patch tests for a micropolar continuum proposed in [21] are generalised to 3D and the elements are tested on an irregular mesh. The finite elements are also tested on two boundary value problems that are important for the experimental determination of the micropolar material parameters, showing the size-effect phenomenon. In Section 5.3, the cylindrical bending of a cantilever beam (also referred to as the higher-order patch test) is analyzed and compared to the analytical solution [4]. Finally, in the last numerical example, presented in Section 5.4, an axisymmetric boundary-value problem consisting of a solid cylinder subject to torsion in two different configurations (two sets of material parameters) is analyzed. In the second configuration, the obtained numerical results are compared against the experimental results given in [10].

\subsection{Force patch test}

We perform the force patch test [31] on a cantilever beam subject to pure tension, as shown in Fig. 2, and check if for an arbitrary number of finite elements in the mesh the exact solution for the state of constant stress is returned. The geometry of the cantilever beam is chosen as $L=5 \mathrm{~m}, h=2 \mathrm{~m}, b=1 \mathrm{~m}$. The free-end of the cantilever beam is subjected to constant axial distributed loading $p_{y}=10 \mathrm{~N} / \mathrm{m}^{2}$, leading to a constant stress field. The constant distributed surface loading is applied through corresponding concentrated nodal forces obtained by integration, which, for a singleelement mesh gives $F=\frac{1}{4} p_{y} b h$, as shown in Fig. 2. At the lefthand end of the cantilever all the displacements in the longitudinal direction are fixed, i.e. $u_{y}(x, 0, z)=0$, while $u_{z}(x, 0,0)=0$, and $u_{x}(0,0,0)=0$ for $x \in[0, b], z \in[0, h]$. The patch test is performed on two regular meshes by equally increasing the number of uniform elements in the $x, y$ and $z$ direction for the chosen material parameters $\mu=1000 \mathrm{~N} / \mathrm{mm}^{2}, \lambda=1000 \mathrm{~N} / \mathrm{mm}^{2}, v=500 \mathrm{~N} / \mathrm{mm}^{2}$, $\alpha=20 \mathrm{~N}, \beta=20 \mathrm{~N}$ and $\gamma=20 \mathrm{~N}$, where the boundary conditions and external loading are correspondingly defined.

It is observed that, even without the matrix modification defined in (26), for a regular mesh the micropolar trilinear hexahedral element with incompatible modes reproduces the analytical results to the highest computer accuracy, which ensures that the element will converge to the exact solution when refining the mesh.

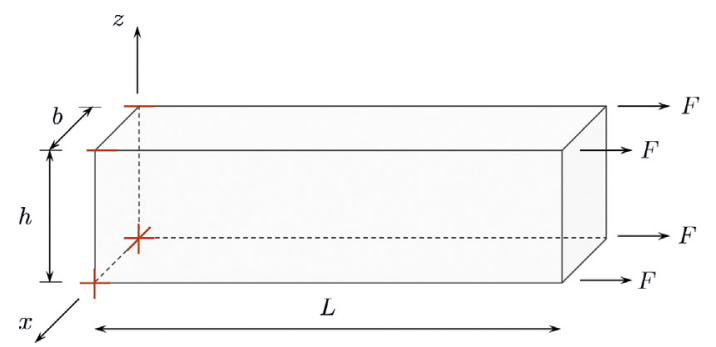

Fig. 2. Cantilever beam subject to constant distributed axial load.

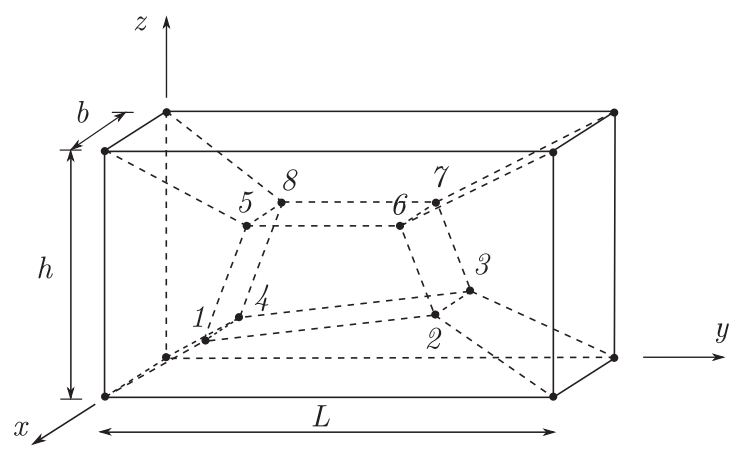

Fig. 3. Finite element mesh for the displacement patch test.

\subsection{Displacement patch tests}

According to Providas and Kattis [21] the patch test for micropolar finite elements should consist of a set of three separate tests. In this work, the tests given in [21] for 2D are generalized to 3D and performed on a cuboid domain with length $L=0.24$, height $h=0.12$, width $b=0.06$ and the internal nodes with the following co-ordinates: $\quad 1=(0.04,0.04,0.02), 2=(0.04,0.18,0.03), \quad 3=$ $(0.02,0.18,0.03), 4=(0.02,0.04,0.02), 5=(0.04,0.08,0.08), \quad 6=$ $(0.04,0.16,0.08), 7=(0.02,0.16,0.08)$ and $8=(0.02,0.08,0.08)$. The domain is discretized with 7 arbitrarily distorted hexahedral finite-elements as shown in Fig. 3. It is important to note that the generalization of Providas and Kattis's tests to 3D is not unique and, in this work one possible generalization of it is presented. The material parameters used are the same as defined in the force patch test.

The patch tests are performed by imposing the displacements and microrotations on the external nodes, while the volume loading (if any) is imposed in the interior of the domain. The element passes a patch test if the internal nodes are capable of reproducing the analytical solution imposed by the boundary conditions.

The first test is the standard patch test of the finite elements in the classical continuum theory, whereby imposing linearly varying displacement and constant microrotation fields via appropriate boundary conditions without any volume and surface loading we obtain the state of constant symmetric stress and strain. The kinematic fields are defined as follows:

$$
\begin{array}{ll}
u_{x}=10^{-3}(x+0.5 y+z), & u_{y}=10^{-3}(x+y+0.5 z), \\
u_{z}=10^{-3}(0.5 x+y+z), & \varphi_{x}=\varphi_{y}=\varphi_{z}=0.25 \cdot 10^{-3},
\end{array}
$$

leading to the following theoretical solution:

$$
\begin{aligned}
& \sigma_{x x}=\sigma_{y y}=\sigma_{z z}=5.0, \sigma_{x y}=\sigma_{y x}=\sigma_{y z}=\sigma_{z y}=\sigma_{x z}=\sigma_{z x}=1.5, \\
& \epsilon_{x x}=\epsilon_{y y}=\epsilon_{z z}=10^{-3}, \epsilon_{x y}=\epsilon_{y x}=\epsilon_{y z}=\epsilon_{z y}=\epsilon_{x z}=\epsilon_{z x}=0.75 \cdot 10^{-3},
\end{aligned}
$$

with all the couple-stress and curvature components equal to zero.

The second test describes the state of constant non-symmetric shear stresses and strains, for which a constant body moment is needed in order to preserve equilibrium. The kinematic fields and body moments are defined as follows:

$$
\begin{array}{ll}
u_{x}=10^{-3}(x+0.5 y+z), & u_{y}=10^{-3}(x+y+0.5 z), \\
u_{z}=10^{-3}(0.5 x+y+z), & \varphi_{x}=\varphi_{y}=\varphi_{z}=0.75 \cdot 10^{-3}, \\
m_{v x}=m_{v y}=m_{v z}=1.0, &
\end{array}
$$

giving the following theoretical solution

$$
\begin{aligned}
& \sigma_{x x}=\sigma_{y y}=\sigma_{z z}=5.0, \quad \sigma_{x z}=\sigma_{y x}=\sigma_{z y}=1.0 \\
& \sigma_{z x}=\sigma_{x y}=\sigma_{y z}=2.0, \quad \epsilon_{x x}=\epsilon_{y y}=\epsilon_{z z}=10^{-3} \\
& \epsilon_{x z}=\epsilon_{y x}=\epsilon_{z y}=0.25 \cdot 10^{-3}, \quad \epsilon_{z x}=\epsilon_{x y}=\epsilon_{y z}=1.25 \cdot 10^{-3},
\end{aligned}
$$


Table 1

Results for Patch test 3 [21] using the Hex8IM element.

\begin{tabular}{|c|c|c|c|c|c|c|c|}
\hline & $u_{x} \times 10^{-4}$ & $u_{y} \times 10^{-4}$ & $u_{z} \times 10^{-4}$ & $\varphi_{x} \times 10^{-4}$ & $\sigma_{x x}$ & $\mu_{x x}$ & $\mu_{x y}$ \\
\hline & 0.604 & 0.699 & 0.698 & 2.102 & 5.013 & 0.019 & -0.039 \\
\hline Exact & 0.600 & 0.700 & 0.700 & 2.100 & 5.000 & 0.020 & -0.040 \\
\hline
\end{tabular}

with all the couple-stress and curvature components again equal to zero. The third test describes the state of constant curvature, whereby imposing linearly varying displacement, microrotation and body-moment fields as well as a constant body-force field we obtain linearly varying stresses and constant couple-stresses. The input is defined as:

$u_{x}=10^{-3}(x+0.5 y+z), u_{y}=10^{-3}(x+y+0.5 z)$,

$u_{z}=10^{-3}(0.5 x+y+z), \varphi_{x}=\varphi_{y}=\varphi_{z}=10^{-3}(0.25+(x-y-z))$,

$p_{v x}=0, p_{v y}=2, p_{v z}=-2, m_{v x}=m_{v y}=m_{v z}=2(x-y-z)$.

giving the following theoretical solution

$$
\begin{aligned}
& \sigma_{x x}=\sigma_{y y}=\sigma_{z z}=5.0, \sigma_{x z}=\sigma_{y x}=\sigma_{z y}=1.5-(x-y-z), \\
& \sigma_{z x}=\sigma_{x y}=\sigma_{y z}=1.5+(x-y-z), \epsilon_{x x}=\epsilon_{y y}=\epsilon_{z z}=10^{-3}, \\
& \epsilon_{x z}=\epsilon_{y x}=\epsilon_{z y}=10^{-3}(0.75-(x-y-z)), \\
& \epsilon_{z x}=\epsilon_{x y}=\epsilon_{y z}=10^{-3}(0.75+(x-y-z)), \\
& \mu_{x x}=0.02, \mu_{y y}=\mu_{z z}=-0.06, \mu_{x y}=\mu_{x z}=\mu_{y z}=\mu_{z y}=-0.04, \\
& \mu_{y x}=\mu_{z x}=0.04, \kappa_{x x}=\kappa_{y x}=\kappa_{z x}=10^{-3}, \\
& \kappa_{y y}=\kappa_{z z}=\kappa_{x y}=\kappa_{x z}=\kappa_{y z}=\kappa_{z y}=-10^{-3} .
\end{aligned}
$$

Providas and Kattis consider the third patch test to be a necessary condition for finite-element convergence even though in this test the shear stresses and strains are linearly varying. However, according to [31], satisfaction of a patch test in which stress distribution is not constant is not considered to be necessary for convergence and, for this reason, we treat this test as a higher-order patch test, analogous to a pure bending test.

All three tests are first performed using the conventional Hex8 finite element and the obtained results correspond to the analytical solution to within the computer accuracy. When analyzing the first two tests using the enhanced finite element Hex8IM it is observed that, for a distorted mesh analyzed here, the matrix modification as presented in (26) is necessary for the element to pass the patch tests. When applying the matrix modification, both tests are satisfied to within the computer accuracy. However, the third test is not satisfied either way and the obtained results are presented in Table 1. Even though the third patch test is not satisfied, we consider that Hex8IM satisfies the convergence criteria since, as argued above, the finite element is able to reproduce exactly any state of constant stress.

\subsection{Pure bending - higher-order patch test}

In an attempt to experimentally validate the micropolar material parameters, Gauthier and Jahsman [4] provided the analytical solution for stresses, displacements and microrotations of a micropolar elastic plate subject to cylindrical bending. Timoshenko and Goodier [43] showed that in three-dimensional classical elasticity, a plate subject to edge moments $M_{z}$ acting per unit length will in general be deformed into an anticlastic shape. When transverse load is applied, the bending deformation occurs not only in the longitudinal direction, but also in the transverse direction, due to the Poisson's effect. This is defined as an anticlastic deformation.
In the work of Gauthier and Jahsman [4] the plate bending problem of length $L$, height $h$ and thickness $b$, shown in Fig. 4, is analysed by assuming lateral boundary conditions which prevent anticlastic distortion, turning it into cylindrical plane-strain bending problem. In other words, the only admissible displacements are $u_{x}(x, y)$ and $u_{y}(x, y)$ and the only admissible microrotation is $\varphi_{z}(x, y)$. Furthermore, Gauthier and Jahsman imposed the requirement that the stresses and couple stresses are functions of $y$ only, leading to a constant stress distribution in the $x$ direction, and a linearly varying distribution in the $y$ direction. Thus, the nonvanishing stresses are $\sigma_{x x}, \sigma_{z z}, \mu_{z x}$ and $\mu_{x z}$.

In the classical elasticity the only way to simulate a concentrated moment $M$ is by applying a linearly varying normal surface traction $p_{s x}=\frac{2 y}{h} p_{0 x}$. In the micropolar elasticity it is possible to model a concentrated moment by using such a traction and/or a constant surface moment $m_{s z}$. Gauthier and Jahsman have shown that in the micropolar elasticity, in order to obtain a state of pure bending, the external moment $M$ has to be applied using both tractions acting on the same side, i.e. $M=b \int_{-\frac{h}{2}}^{\frac{h}{2}}\left(y p_{s x}+m_{s z}\right) d y$ which are defined as

$p_{s x}=\sigma_{x x}=-\frac{1}{1+(1-n) \delta} \frac{M}{W_{z}} \frac{2 y}{h}$,

$m_{s z}=\mu_{z x}=\frac{(1-n) \delta}{1+(1-n) \delta} \frac{M}{A}$,

where $n$ is Poisson's coefficient and $\delta=24\left(l_{b} / h\right)^{2}$. Therefore, a unique relationship between the external loads is given as

$\frac{m_{s z}}{p_{0 x}}=\frac{1}{h} \frac{(\lambda+2 \mu)(\beta+\gamma)}{2 \mu(\lambda+\mu)} \equiv \frac{h}{6}(1-n) \delta$.

Obviously, for a material with vanishing characteristic length $\left(l_{b} \rightarrow 0\right)$ the state of pure bending may not be achieved if the surface moment loading is present, while for a general micropolar material such a state is only possible when $m_{s z}$ and $p_{0 x}$ are given in the proportion defined above resulting in $M=p_{0 x} W_{z}+m_{s z} A$ with $A=b h$ and $W_{z}=b h^{2} / 6$ (this is sometimes misinterpreted in the literature where the external loading is applied using only one of the surface tractions, e.g. [44]). The non-vanishing displacement and rotation fields are

$\varphi_{z}=\frac{1}{1+(1-n) \delta} \frac{M x}{b D}, \quad u_{x}=-\frac{1}{1+(1-n) \delta} \frac{M x y}{b D}$,

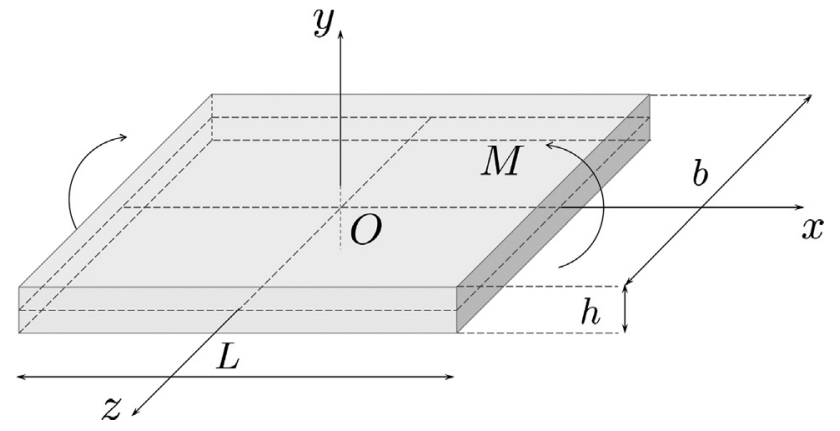

Fig. 4. Bending of a plate. 
$u_{y}=\frac{1}{2} \frac{1}{1+(1-n) \delta} \frac{M}{b D}\left(x^{2}+\frac{n}{1-n} y^{2}\right)$

where $D=\frac{E h^{3}}{12\left(1-n^{2}\right)}$ represents the flexural rigidity. With respect to the classical-elasticity solution, all results are obviously multiplied by the factor $\frac{1}{1+(1-n) \delta}$ leading to an increased bending stiffness, depending on the value of the characteristic length $l_{b}$. In other words, compared to the classical solution where the bending resistance is proportional to the height of the specimen squared, the bending stiffness increases when the value of the material characteristic length $l_{b}$ is increased. The size-effect becomes significant when the material characteristic length gets close to the beam's height, i.e. $l_{b} \rightarrow h$. On the other hand, as $l_{b} \rightarrow 0$, the result tends to the classical-elasticity solution.

In order to test the accuracy of the hexahedral element enhanced with incompatible modes, a cantilever beam of length $L=10 \mathrm{~m}$, height $h=2 \mathrm{~m}$ and thickness $b=1 \mathrm{~m}$, shown in Fig. 5 submitted to cylindrical bending is analyzed. The problem is solved while varying the value of the characteristic length $l_{b}=\sqrt{\frac{\beta+\gamma}{G}}$, $l_{b} \in[0.0,1.8]$ to capture the size-effect. The resultant bending moment $M=20 \mathrm{~N} \mathrm{~m}$ is applied through a linearly varying surface loading and a constant surface moment loading in the defined proportion, as defined in Table 2. The distributed loading is applied through corresponding concentrated nodal forces and moments obtained by integration as defined by (11)-(13).

The engineering material parameters are taken to be equal to $E=1500 \mathrm{~N} / \mathrm{m}^{2}$ and $n=0.25$ which give the Lamé constants $\mu=600 \mathrm{~N} / \mathrm{m}^{2}$ and $\lambda=600 \mathrm{~N} / \mathrm{m}^{2}$. The parameter $v$ is chosen to be equal to $v=200 \mathrm{~N} / \mathrm{m}^{2}$, corresponding to $N=0.5$, but in this example it can have an arbitrary value, since the problem does not induce any non-symmetry. The remaining engineering parameters are chosen as $\psi=0$ and $l_{t}=0.1$, but, since they do not affect the solution, they can also have arbitrary values. Along the left-hand edge of the specimen all the horizontal displacements and microrotations are restrained, i.e. $u_{x}(0, y, z)=\varphi_{x}(0, y, z)=$

Table 2

External loading depending on the value of $l_{b}$.

\begin{tabular}{cccc}
\hline$l_{b}$ & $\beta+\gamma$ & $p_{0}$ & $m_{s z}$ \\
\hline 0 & 0.0 & 30.000000000000000 & 0.000000000000000 \\
0.1 & 24.0 & 28.708133971291860 & 0.430622009569378 \\
0.3 & 216.0 & 21.352313167259780 & 2.882562277580070 \\
0.6 & 864.0 & 11.450381679389320 & 6.183206106870228 \\
1.2 & 3456.0 & 4.010695187165778 & 8.663101604278070 \\
1.8 & 7776.0 & 1.925545571245185 & 9.358151476251610 \\
\hline
\end{tabular}

$\varphi_{y}(0, y, z)=\varphi_{z}(0, y, z)=0$, for $y \in[0, h]$ and $z \in[0, b]$. The vertical displacement at the left-hand edge is restrained only at the cantilever axis, i.e. $u_{y}\left(0, \frac{h}{2}, z\right)=0$ for $z \in[0, b]$. Furthermore, the cylindrical bending of the specimen is accomplished by additionally restraining the displacements in the $z$ direction along the whole cantilever, i.e. $u_{z}(x, y, z)=0$ for $x \in[0, L], y \in[0, h]$ and $z \in[0, b]$.

The problem is solved using a mesh of two hexahedral elements as shown in Fig. 5. The results for the vertical displacement and microrotation $u_{y}$ and $\varphi_{z}$ at node $P$ and the stress $\sigma_{x x}$ at the Gauss point with coordinates $G P=(7.88675,0.211325,0.788675)$ obtained by Hex8 and Hex8IM are compared to the analytical solution and shown in Table 3 and Fig. 6.

From the obtained results we can see that Hex8IM reproduces the analytical solution to within the computer accuracy, while the conventional element with Lagrange interpolation Hex8 shows very poor results, especially for smaller micropolar effects. The improvement due to the incompatible modes is highly significant. Even with a very coarse mesh, the analytical solution of this higher-order patch test is precisely reproduced.

\subsection{Micropolar solid cylinder under torsional load}

An axisymmetric solid micropolar cylinder subject to pure torsion shown in Fig. 7 is analyzed in this example. Gauthier and Jahsman derived the analytical solution for a cylindrical specimen of height $c$ and cross-section radius $a$ in the cylindrical coordinate system $(r, \theta, z)$ subject to torsional load [4]. The stresses and couple stresses are axisymmetric, and independent of $z$. Furthermore, all non-vanishing variables are independent of the angle $\theta$. By further imposing a traction-free surface for $r=a$, prescribing the appropriate resultant torque $T$ on end surfaces $z=0$ and $z=c$ and taking into account the compatibility conditions, the analytical solution for stresses, displacements and microrotations are obtained.

The first comprehensive numerical study of the problem is presented in [45], where linear beam finite elements are tested for a range of micropolar material parameters and the obtained results are compared to the analytical solution. Furthermore, in [44] three-dimensional non-linear finite elements are developed and their performance is tested by modeling this linear-elastic problem. A good agreement between the numerical and analytical results is shown in both references. However, as in the purebending case from Section 5.3, Gauthier and Jahsman have shown that in the micropolar theory the state of axisymmetric torsion of a circular cylinder can be achieved only by applying both a normal surface traction $p_{s \theta}$ and a surface moment traction $m_{s z}$. In other words, to correctly model the problem in $3 \mathrm{D}$, the external torque $T$ should be applied as

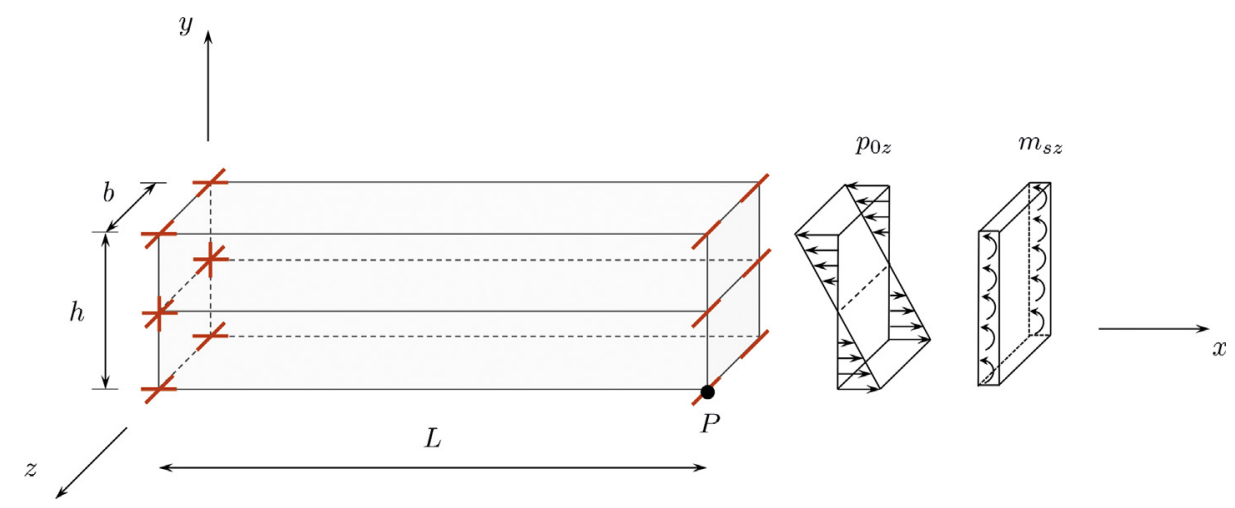

Fig. 5. Cantilever beam subject to pure bending. 
Table 3

Results obtained using two hexahedral elements with eight nodes (Hex8 and Hex8IM), $2 \times 2 \times 2$ integration points, A = Analytical, $\mathrm{N}=\mathrm{Numerical}$.

\begin{tabular}{|c|c|c|c|c|c|c|c|c|}
\hline Element & $l_{b}$ & $\beta+\gamma$ & $\begin{array}{l}\text { A } \\
u_{y}\end{array}$ & $\begin{array}{l}\mathrm{N} \\
u_{y}\end{array}$ & $\begin{array}{c}\mathrm{A} \\
\varphi_{z} \\
\end{array}$ & $\begin{array}{c}\mathrm{N} \\
\varphi_{z} \\
\end{array}$ & $\begin{array}{c}\mathrm{A} \\
\sigma_{x x, G P} \\
\end{array}$ & $\begin{array}{c}\mathrm{N} \\
\sigma_{x x, G P} \\
\end{array}$ \\
\hline $\begin{array}{c}\text { Hex8 } \\
\text { Hex8IM }\end{array}$ & 0.0 & 0.0 & 0.94063 & $\begin{array}{l}0.06910 \\
0.94063\end{array}$ & 0.18750 & $\begin{array}{l}0.01260 \\
0.18750\end{array}$ & 23.6603 & $\begin{array}{c}1.9684 \\
23.6603\end{array}$ \\
\hline $\begin{array}{c}\text { Hex8 } \\
\text { Hex8IM }\end{array}$ & 0.1 & 24.0 & 0.90012 & $\begin{array}{l}0.06892 \\
0.90012\end{array}$ & 0.17943 & $\begin{array}{l}0.01269 \\
0.17943\end{array}$ & 22.6414 & $\begin{array}{c}1.9503 \\
22.6414\end{array}$ \\
\hline $\begin{array}{c}\text { Hex8 } \\
\text { Hex8IM }\end{array}$ & 0.3 & 216.0 & 0.66948 & $\begin{array}{l}0.06740 \\
0.66948\end{array}$ & 0.13345 & $\begin{array}{l}0.01296 \\
0.13345\end{array}$ & 21.3523 & $\begin{array}{c}1.8345 \\
21.3523\end{array}$ \\
\hline $\begin{array}{c}\text { Hex8 } \\
\text { Hex8IM }\end{array}$ & 0.6 & 864.0 & 0.35902 & $\begin{array}{l}0.06203 \\
0.35902\end{array}$ & 0.07157 & $\begin{array}{l}0.01261 \\
0.07157\end{array}$ & 11.4504 & $\begin{array}{c}1.5997 \\
11.4504\end{array}$ \\
\hline $\begin{array}{c}\text { Hex8 } \\
\text { Hex8IM }\end{array}$ & 1.2 & 3456.0 & 0.12575 & $\begin{array}{l}0.04624 \\
0.12575\end{array}$ & 0.02507 & $\begin{array}{l}0.00977 \\
0.02507\end{array}$ & 3.1631 & $\begin{array}{l}1.1436 \\
3.1631\end{array}$ \\
\hline $\begin{array}{c}\text { Hex8 } \\
\text { Hex8IM }\end{array}$ & 1.8 & 7776.0 & 0.06037 & $\begin{array}{l}0.03234 \\
0.06037\end{array}$ & 0.01204 & $\begin{array}{l}0.00691 \\
0.01204\end{array}$ & 1.5186 & $\begin{array}{l}0.7904 \\
1.5186\end{array}$ \\
\hline
\end{tabular}

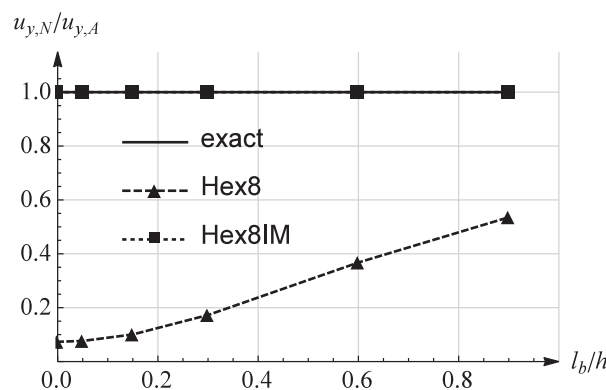

(a) Normalised end-node vertical displacements

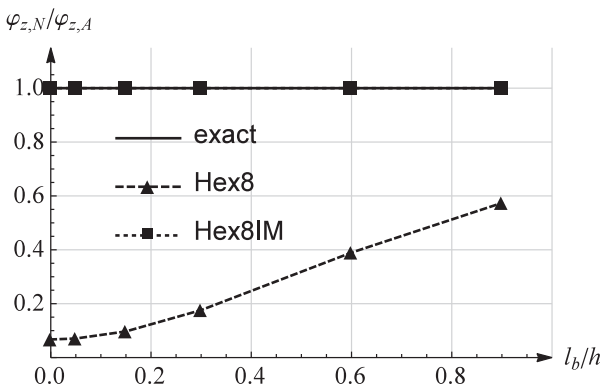

(b) Normalised end-node microrotation

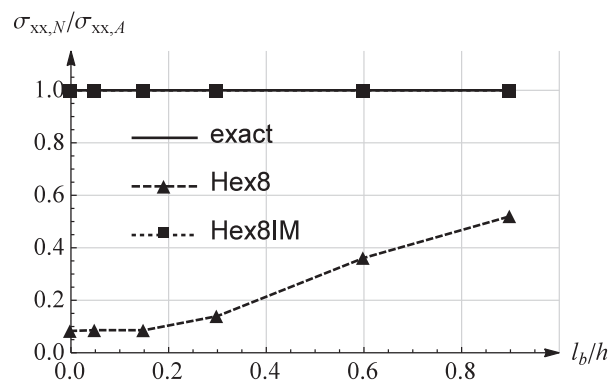

(c) Normalised stresses in Gauss point

Fig. 6. Cantilever beam subject to pure bending - results for Hex8 and Hex8IM.

$\int_{A}\left(r p_{s \theta}+m_{s z}\right) d A=T$,

where $A=r^{2} \pi$ is the cylinder cross-section area, $r$ is the variable in the radial direction, $p_{s \theta}$ is the tangential surface loading and $m_{s z}$ is the moment surface loading, as presented in [4]. The Neumann boundary condition is then $2 \pi \int_{0}^{a}\left(r^{2} \sigma_{\theta z}+r \mu_{z z}\right) d r=T$ where $a$ represents the cross-section radius and $\sigma_{\theta z}$ and $\mu_{z z}$ represent the stress and couple-stress components, respectively, with the first index denoting the direction and the second index denoting the surface normal. According to the analytical solution, both $\sigma_{\theta z}$ and $\mu_{z z}$ are described by the modified Bessel functions of the first kind $I_{n}(r)$ [46] depending on $r$ and multiplied by constants of integration $C_{1}$ and $C_{9}$, as follows:

$\sigma_{\theta z}=p_{s \theta}=\mu C_{1} r+2 v C_{9} I_{1}(p r)$,

$\mu_{z z}=m_{s z}=\alpha p C_{9} I_{0}(p r)+2 \beta C_{1}$, where

$p=\sqrt{\frac{4 v}{\alpha+2 \beta}}$

$C_{9}=\frac{T}{2 \pi a^{2}}\left[\left(\frac{\mu a^{2}}{4 \beta}+1.5\right)(\alpha+2 \beta) p I_{0}(p a)-\left(\frac{\mu a^{2}}{4 \beta}+2\right) \frac{2 \beta}{a} I_{1}(p a)\right]^{-1}$

and $C_{1}=2 C_{9}\left(\frac{\alpha+2 \beta}{2 \beta} p I_{0}(p a)-\frac{1}{a} I_{1}(p a)\right), I_{0}$ and $I_{1}$ being the modified Bessel functions of the first kind. In other words, the distribution of the external loading is directly dependent on material parameters. The remaining non-vanishing variables are $\sigma_{z \theta}, \mu_{r r}, \mu_{\theta \theta}, u_{\theta}, \varphi_{r}$ and $\varphi_{z}$, where the displacement and rotation fields are defined as

$u_{\theta}=C_{1} r z, \quad \varphi_{r}=-\frac{C_{1} r}{2}+C_{9} I_{1}(p r), \quad \varphi_{z}=C_{1} z$,

$u_{\theta}$ being linear in $r$ and $z$ and $\varphi_{z}$ linear in $z$ as in the classical elasticity. Since $u_{z}$ vanishes, no warping of surfaces is predicted. 


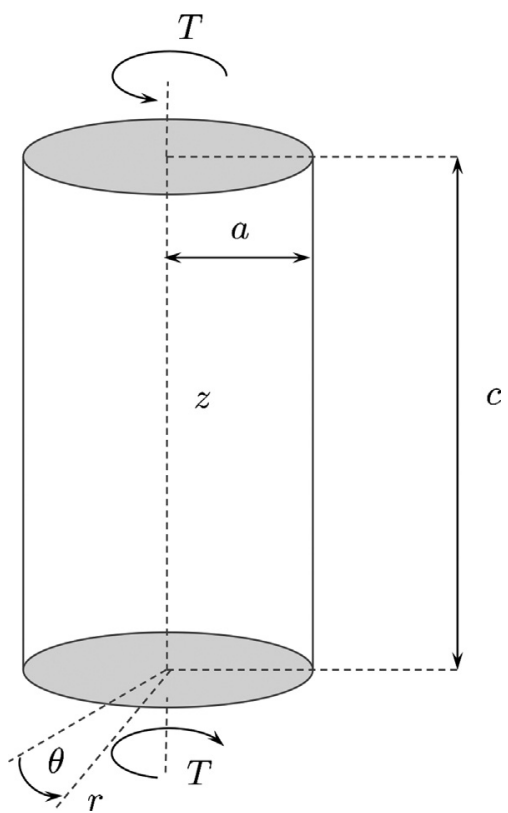

Fig. 7. Solid cylinder in torsion.

In order to relate the classical and micropolar torsional problem, Gauthier and Jahsman introduce a parameter $\Omega$ which defines the ratio of the micropolar torsional rigidity to the classical torsional rigidity $J=\frac{G \pi a^{4}}{2}$. The ratio $\Omega$ is given as a function of engineering micropolar material parameters as:

$\Omega=1+\frac{6}{a^{2}} l_{t}^{2} \frac{1-\frac{4}{3} \psi \chi}{1-\psi \chi}, \quad$ where $\quad \chi=\frac{I_{1}(p a)}{p a I_{0}(p a)} \quad$ and

$p=\sqrt{\frac{2 \psi N^{2}}{l_{t}^{2}\left(1-N^{2}\right)} .}$
It can be seen that for the limiting case $l_{t} \rightarrow 0$ the micropolar rigidity approaches the classical-elasticity value, since the ratio $\Omega \rightarrow 1$. On the other hand, for the limiting case $\psi \rightarrow 0$, the ratio of micropolar rigidity approaches its maximum value of $\Omega=1+6\left(\frac{l_{t}}{a}\right)^{2}$. In general, as the characteristic length approaches the specimen radius, the micropolar rigidity increases and it can be as many as seven times bigger than the classical rigidity.

In the first part of this analysis the problem is solved using both Hex 8 and Hex8IM elements and the finite elements are tested by comparing the numerical results against the analytical solution. The radius of the cylinder is taken as $a=0.2 \mathrm{~mm}$, its height is $c=1 \mathrm{~mm}$, and it is subjected to a resultant torque $T=1 \mathrm{Nmm}$. The chosen material parameters are $\mu=10,500 \mathrm{~N} / \mathrm{mm}^{2}$, $\lambda=157,500 \mathrm{~N} / \mathrm{mm}^{2}, \quad v=3500 \mathrm{~N} / \mathrm{mm}^{2}, \alpha=0 \mathrm{~N}, \quad \beta=105 \mathrm{~N}$ and $\gamma=-105 \mathrm{~N}$, which corresponds to the following engineering material parameters: $E=30843.8 \mathrm{~N} / \mathrm{mm}^{2}, n=0.46875, N=0.5, l_{b}=0$ $\mathrm{mm}, l_{t}=0.1 \mathrm{~mm}, \psi=1.0$. Since $\alpha=0$, the first term in $(45)_{2}$ vanishes, i.e. $m_{s z}$ becomes constant and we obtain the external loading as shown in Fig. 8.

The surface traction is in general a non-linear function, as shown in Fig. 8a. By extracting an initial part of that diagram (the detail in Fig. 8a), we can see that as $r$ gets smaller, the shape of $p_{s \theta}$ approaches a linear function, as blown up in Fig. $8 \mathrm{~b}$. We can thus say that $p_{s \theta}$ is nearly linear for $r \in[0, a]$ and the resultant torque $T$ is modelled as a linearly varying surface loading $p_{s \theta}$ where $p_{s \theta}(0, \theta, c)=0 \mathrm{~N} / \mathrm{mm}^{2}, \quad p_{s \theta}(r, \theta, c)=43.93046972 \mathrm{~N} / \mathrm{mm}^{2}$, $\theta \in[0,2 \pi]$, along with a constant distributed moment surface loading $m_{s z}=3.636829403 \mathrm{~N} \mathrm{~mm} / \mathrm{mm}^{2}$ shown in Fig. 8c. Along the bottom side of the cylinder $(z=0)$ all the displacements and microrotation $\varphi_{z}$ are restrained. The problem is solved for two different mesh densities, with 24 and 144 elements, as shown in Fig. 9a and b, respectively.

The results obtained by Hex8 and Hex8IM for microrotation $\varphi_{z}$ along the cylinder axis $z$ for $r=a$, and microrotation $\varphi_{r}$ and dis-

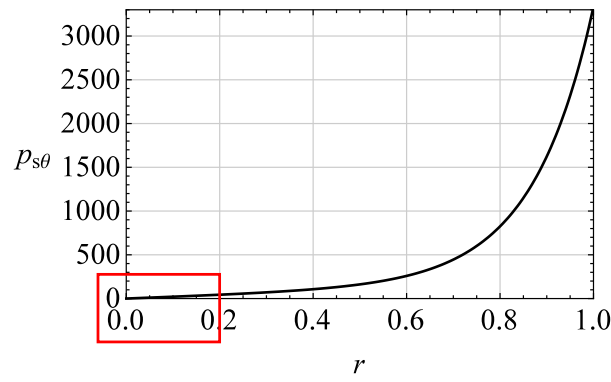

(a) $p_{s \theta}$ for $r \in[0,1.0]$

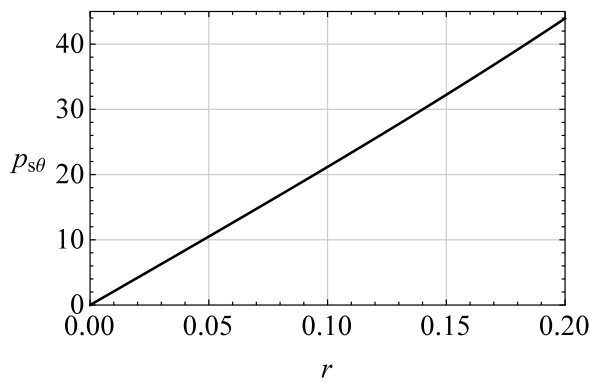

(b) $p_{s \theta}$ for $r \in[0, a]$

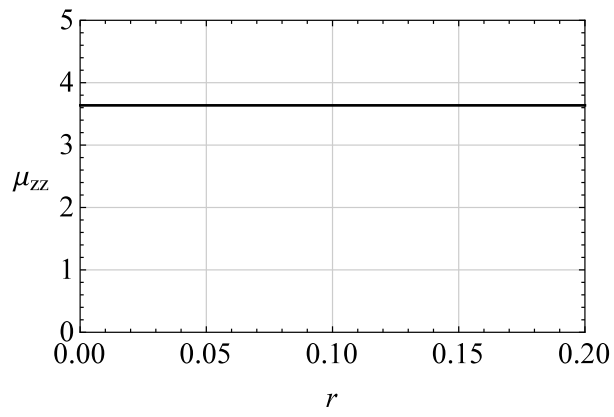

(c) $m_{s z}$ for $r \in[0, a]$

Fig. 8. Distribution of the external loading. 


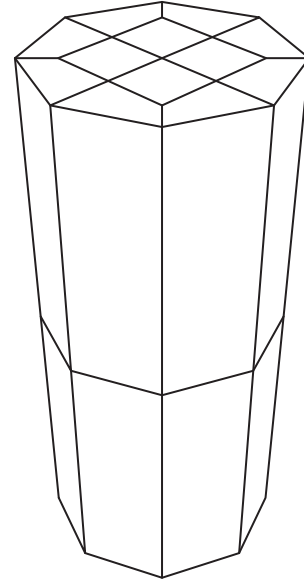

(a) 24 elements

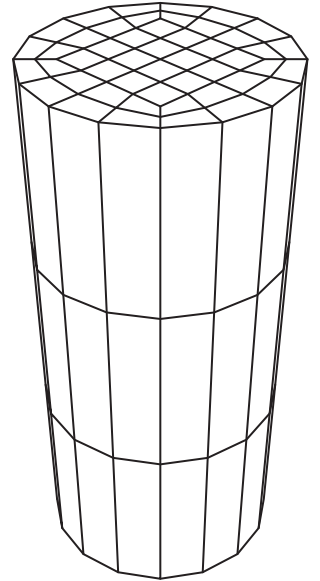

(b) 144 elements

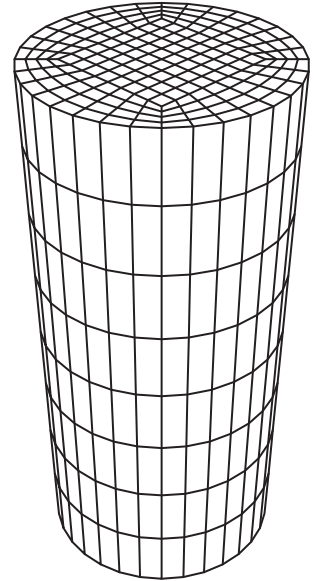

(c) 1536 elements

Fig. 9. Finite element mesh for the axisymmetric problem

placement $u_{\theta}$ at the upper edge $(z=c)$ along $r$ are compared against the analytical solution, as shown in Figs. 10-12.

We can see that both the Lagrange element Hex8 and the enhanced element Hex8IM follow the analytical solution, and the numerical results are in good agreement with the analytical solution even for a coarse mesh. The numerical analysis correctly predicts the linear distribution of the axial microrotation component $\varphi_{z}$ and the displacement component $u_{\theta}$. The results for the radial microrotation component $\varphi_{r}$ correctly follow the analytical trend. Because of the presence of the characteristic length for torsion, the rigidity of this micropolar cylinder is 2.19 times larger than expected classically. However, the enhancement due to incompatible modes does not improve the convergence rate.

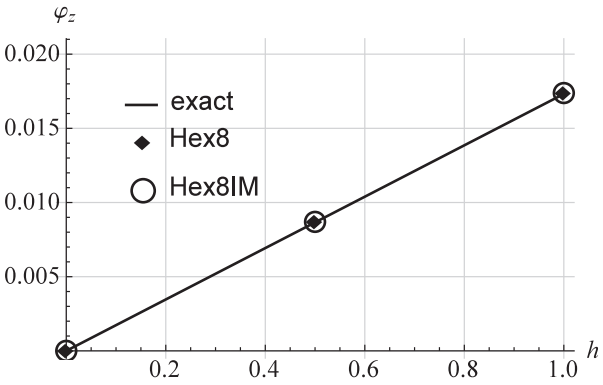

(a) 24 elements
As in the cylindrical bending example from Section 5.3, it is important to note that when the resultant moment $T$ is modeled as a constant distributed moment surface loading $T=\int_{A} m_{s z} d A$, or a linearly varying surface loading $T=\int_{A} r p_{s \theta} d A$ only, an axisymmetric torsion state is not obtained. Consequently, such a problem does not converge to the analytical solution given in [4] but to another different solution. Such a problem is analyzed in [44].

In the second part of this numerical example the results of the numerical analysis are compared with the experiments performed on a micropolar material. The first successful attempt to experimentally validate all six micropolar material parameters is conducted by Lakes [10] who has studied experimentally the sizeeffect phenomenon, which is analytically predicted to occur in tor-

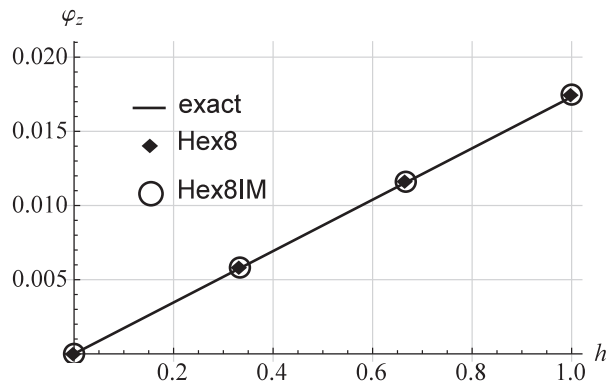

(b) 144 elements

Fig. 10. Distribution of $\varphi_{z}$ along $z$ - results for Hex8 and Hex8IM for different mesh densities.

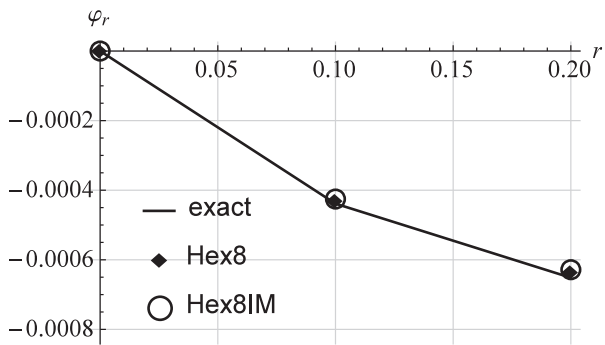

(a) 24 elements

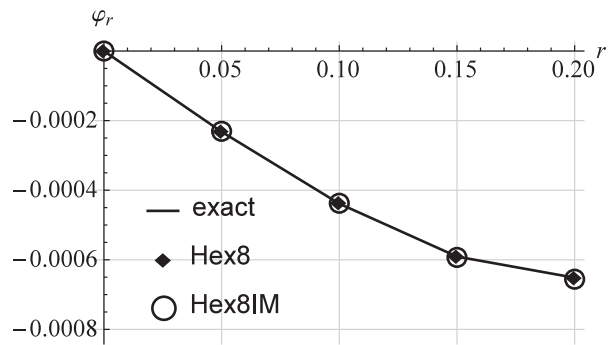

(b) 144 elements

Fig. 11. Distribution of $\varphi_{r}$ along $r$ - results for Hex8 and Hex8IM for different mesh densities. 


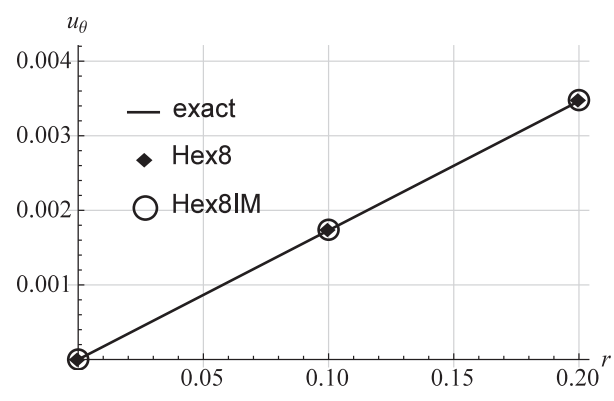

(a) 24 elements

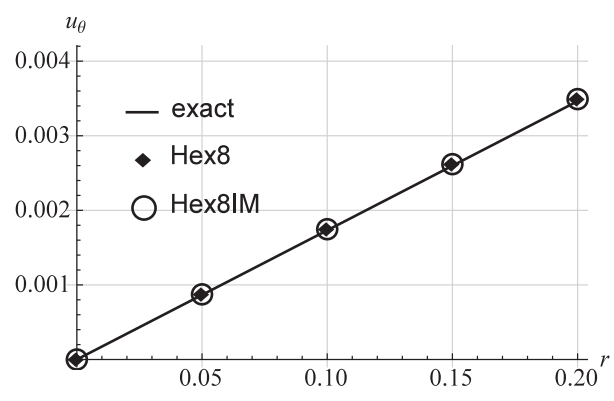

(b) 144 elements

Fig. 12. Distribution of $u_{\theta}$ along $r$ for $z=c$ - results for Hex8 and Hex8IM for different mesh densities.

sion and bending [4]. His study consists of a set of quasi-static torsion and bending tests performed on circular cylinder specimens and dynamic tests performed on rectangular bars made of lowdensity polymeric foam. A characteristic dimension of the specimens is taken to be small enough for the size effect to be observable, approaching the value of the material characteristic length (diameters $13 \mathrm{~mm}, 20 \mathrm{~mm}, 28 \mathrm{~mm}, 35 \mathrm{~mm}$ and $40 \mathrm{~mm}$ with the length-to-diameter ratio $c / d=5$ ). The end-point torsional rotation $\theta$ is measured for a given torque value and the resulting torsional rigidity is computed from $J=\frac{T c}{\theta}$. The results of $\frac{J}{d^{2}}$ against $d^{2}$ obtained in this way in [10] are reproduced as dots in Fig. 13. Analytically, on the other hand, the micropolar torsional rigidity follows from $(46)_{3}$ as

$J=\frac{T}{C_{1}}=\pi a^{2} \frac{\left(\frac{\mu a^{2}}{2 \beta}+3\right)\left(\frac{\alpha}{2}+\beta\right) p a I_{0}(p a)-\left(\frac{\mu a^{2}}{2 \beta}+4\right) \beta I_{1}(p a)}{\left(1+\frac{\alpha}{2 \beta}\right) p a I_{0}(p a)-I_{1}(p a)}$,

with $a=\frac{d}{2}$ and $p=2 \sqrt{\frac{v}{\alpha+2 \beta}}$, i.e. it is a function of the micropolar material parameters $\mu, v, \alpha, \beta$ and the cross-section radius $a$. Lakes has determined these material parameters [10] by drawing the best-fit curve to the experimental results, plotted using a solid line in Fig. 13. The micropolar engineering parameters $G, l_{t}, N$, and $\psi$ can then be obtained from Eq. (10). Lakes refers to this approach as the method of size effects which makes use of the analytical solution [4] to describe the dependence of rigidity upon size. For the case of the polymeric-foam specimens, the experimental data are fitted well by $G=0.6 \mathrm{~N} / \mathrm{mm}^{2}, \psi=1.5, l_{t}=3.8 \mathrm{~mm}$ and $N=0.3$. The remaining engineering parameters are obtained from the tension and bending test and are equal to $n=0.07$ and $l_{b}=5 \mathrm{~mm}$. The corresponding continuum material parameters are $\mu=0.6 \mathrm{~N} / \mathrm{mm}^{2}$, $\lambda=0.0976744 \mathrm{~N} / \mathrm{mm}^{2}, \quad v=0.0593407 \mathrm{~N} / \mathrm{mm}^{2}, \quad \alpha=-5.776 \mathrm{~N}$, $\beta=8.664 \mathrm{~N}$ and $\gamma=51.336 \mathrm{~N}$. Let us note that here the restriction on positive definiteness of the strain energy is not strictly satisfied since $3 \alpha+2 \beta=0$. The dashed line in Fig. 13 represents the theoretical solution in the classical-elasticity theory, $\frac{J}{d^{2}}=\frac{1}{32} \pi G d^{2}$.

In our numerical model proper external loading should be applied as argued earlier. For the micropolar parameters given and a unit torque moment $T=1 \mathrm{~N} \mathrm{~mm}$, the distributed surface loading is represented by a quasi-linearly varying surface loading $p_{s \theta}$ and, since $\psi \neq 0$, a non-constant distributed surface moment loading $m_{s z}$. For the specimen with $d=13 \mathrm{~mm}$, this is shown in Fig. 14.

By analyzing the definition of the resultant torque $T$ from Eq. (44), we can see from Fig. 14 that for the specimen with diameter $d=13 \mathrm{~mm}$ the contribution of the distributed surface load $m_{s z}$ in the resultant unit torque moment is $25.15 \%$ while the contribution of $p_{s \theta}$ is $74.85 \%$. Furthermore, the contribution of the constant part of the surface moment loading, having the value of $m_{s z}=0.001420949939 \mathrm{~N} \mathrm{~mm} / \mathrm{mm}^{2}$ is $18.86 \%$ while the contribution of the non-linear part of the surface moment loading is $6.28 \%$, all computed using the Wolfram Mathematica package.

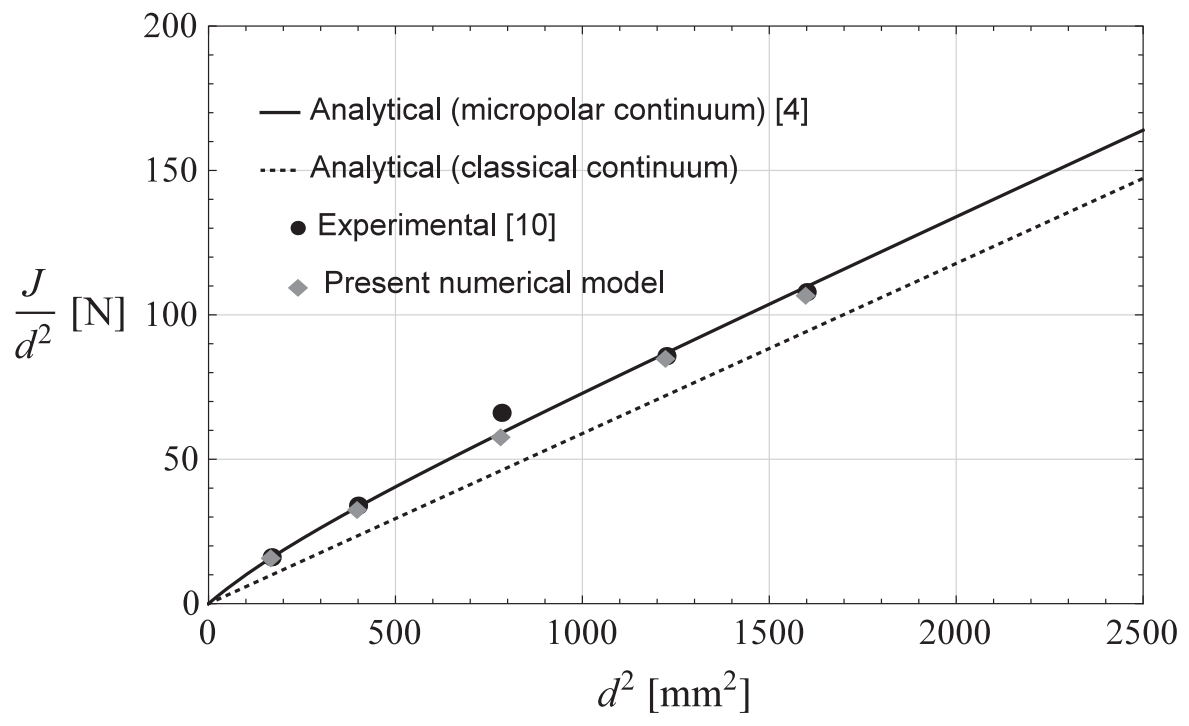

Fig. 13. Analytical, experimental and numerical representation of a size-effect behaviour of a polymeric foam. 


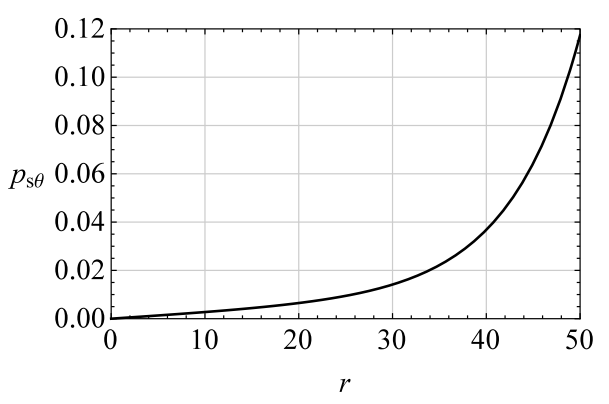

(a) $p_{s \theta}$ for $r \in[0,50.0]$

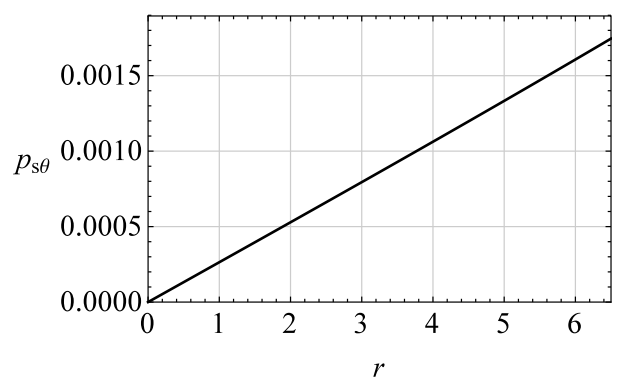

(b) $p_{s \theta}$ for $r \in[0, a]$

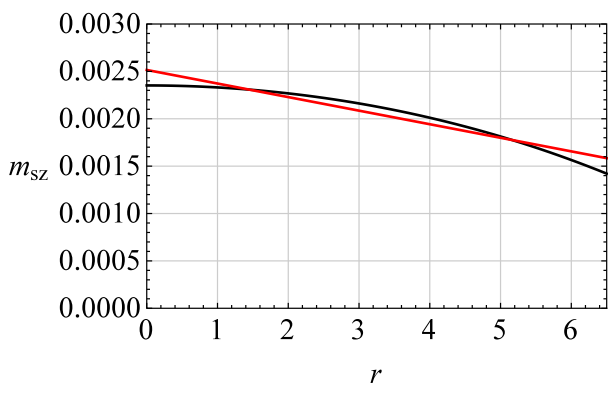

(c) $m_{s z}$ for $r \in[0, a]$

Fig. 14. Distribution of the external loading for the specimen with $d=13 \mathrm{~mm}$.

Thus, the applied moment loading is simplified to a sum of the constant part of the moment surface loading equal to $m_{s z}=0.001420949939 \mathrm{~N} \mathrm{~mm} / \mathrm{mm}^{2}$ and a radially linear distribution obtaining the value of $0.001094166286 \mathrm{~N} \mathrm{~mm} / \mathrm{mm}^{2}$ for $r=0$ and $0.0001634 \mathrm{~N} \mathrm{~mm} / \mathrm{mm}^{2}$ for $r=a$. This distribution is shown in Fig. 14c, represented by the straight line such that the areas under the analytical result (curved line) and the approximated result (straight line) are the same. Finally, the surface loading is applied as approximately linearly varying to obtain the value $p_{s \theta}=0.001745546194 \mathrm{~N} / \mathrm{mm}^{2}$ for $r=a$. The external loading for the remaining specimens is applied analogously, with the corresponding values obtained from (45). For the remaining specimens, the contribution of the distributed surface load $m_{s z}$ in the resultant unit torque moment is decreasing by increasing the specimen diameter.

The problem is solved using Hex8IM elements for a fine mesh of 1536 elements shown in Fig. 9c), and the obtained numerical results for $u_{\theta}$ at point $P(a, 0, c)$ for all specimens are introduced into the definition of the rigidity $J=\frac{T a c}{u_{\theta}}$ and plotted as diamonds in Fig. 13. Even with the applied external loading simplified as described, very good agreement with the experiments conducted in [10] is achieved. Finally, in this example it is observed that, according to the experiments shown in Fig. 13, the rigidity of the specimen with $d=13 \mathrm{~mm}$ is approximately $60 \%$ higher, while the rigidity of the specimen with $d=20 \mathrm{~mm}$ is approximately $16.5 \%$ higher than predicted by the classical-elasticity theory, which is now also numerically proven.

\section{Conclusion}

In the framework of the micropolar continuum theory, the performance of a 1st order hexahedral finite element enhanced with incompatible modes is analyzed. The element is tested through four numerical examples and compared to the conventional hexahedral element interpolated using standard Lagrange interpolation. The motivation for the choice of the numerical examples is found in the available analytical solutions for various boundary value problems, which are significant for the experimental verification of the micropolar material parameters. After assuring convergence of the enhanced finite element by passing the patch test for constant stresses, a cylindrical bending test is performed, where it is shown that the enhancement due to incompatible modes is significant. The resulting element is able to correctly reproduce the analytical solution, while the conventional element gives poor results. Finally, pure torsion tests on circular cylinders of different geometry are performed and the numerical analysis is put into the context of the experimental analysis of a polymeric foam. It is shown that the finite element correctly describes the size-effect phenomenon predicted analytically and observed experimentally. An excellent agreement between theory, experiments and the numerical analysis is achieved. However, it is observed that the enhancement due to incompatible modes does not contribute to a higher convergence rate in the pure torsion tests, compared to the conventional finite element. The reason is simple, since the incompatible modes are not needed for pure torsion (contrary to the pure bending test), given corresponding displacement linear variation.

It can be concluded that Hex8IM highly reduces the computational cost in the cylindrical bending problem and correctly predicts the size-effect phenomenon in bending and torsion. Owing to that, the use of the presented element as a part of the numerical validation of the experimental procedure can be considered to be highly efficient.

\section{Acknowledgement}

The research presented in this paper has been financially supported by the Croatian Science Foundation grants Configurationdependent Approximation in Non-linear Finite-element Analysis of Structures (HRZZ-IP-11-2013-1631), Young Researchers' Career Development Project - Training of Doctoral Students and the French Government scholarship. Professor Adnan Ibrahimbegović has 
been supported by IUF - Institut Universitaire de France. These supports are gratefully acknowledged.

\section{References}

[1] Nowacki W. Theory of micropolar elasticity. Vienna: Springer-Verlag; 1972.

[2] Eringen AC. Microcontinuum field theories: I. Foundations and solids. New York: Springer-Verlag; 2012.

[3] Cosserat E, Cosserat F. Théorie des corps déformables, Herman, Paris; 1909.

[4] Gauthier R, Jahsman WE. A quest for micropolar elastic constants. J Appl Mech 1975;42(2):369-74. https://doi.org/10.1115/1.3423583.

[5] Yang JFC, Lakes RS. Transient study of couple stress effects in compact bone: torsion. J Biomech Eng 1981;103(4):275-9. https://doi.org/10.1115 $\underline{1.3138292}$.

[6] Yang JFC, Lakes RS. Experimental study of micropolar and couple stress elasticity in compact bone in bending. J Biomech 1982;15(2):91-8. https://doi. org/10.1016/0021-9290(82)90040-9.

[7] Lakes RS, Nakamura S, Behiri JC, Bonfield W. Fracture mechanics of bone with short cracks. J Biomech 1990;23(10):967-75. https://doi.org/10.1016/00219290(90)90311-P.

[8] Anderson WB, Lakes RS. Size effects due to Cosserat elasticity and surface damage in closed-cell polymethacrylimide foam. J Mater Sci 1994;29 (24):6413-9. https://doi.org/10.1007/BF00353997.

[9] Rueger Z, Lakes RS. Cosserat elasticity of negative Poisson's ratio foam: experiment. Smart Mater Struct 2016;25(5):054004. https://doi.org/10.1088/ 0964-1726/25/5/054004.

[10] Lakes RS. Size effects and micromechanics of a porous solid. J Mater Sci 1983;18:2572-80. https://doi.org/10.1007/BF00547573.

[11] Lakes RS. Experimental microelasticity of two porous solids. Int J Solids Struct 1986;22(1):55-63. https://doi.org/10.1016/0020-7683(86)90103-4.

[12] Chen CP, Lakes RS. Holographic study of conventional and negative Poisson's ratio metallic foams: elasticity, yield and micro-deformation. J Mater Sci 1991;26(20):5397-402. https://doi.org/10.1007/BF02403936.

[13] Bažant Z, Christensen M. Analogy between micropolar continuum and grid frameworks under initial stress. Int J Solids Struct 1972;8(3):327-46. https:/l doi.org/10.1016/0020-7683(72)90093-5.

[14] Wang XL, Stronge WJ. Micropolar theory for two-dimensional stresses in elastic honeycomb. Proc Roy Soc A, Math, Phys Eng Sci 1999;455 (1986):2091-116. https://doi.org/10.1098/rspa.1999.0394.

[15] Mora RJ, Waas AM, Arbor A. Evaluation of the Micropolar elasticity constants for honeycombs. Acta Mech 2007;192:1-16. https://doi.org/10.1007/s00707007-0446-8.

[16] Besdo D. Towards a Cosserat-theory describing motion of an originally rectangular structure of blocks. Arch Appl Mech 2010;80(1):25-45. https:// doi.org/10.1007/s00419-009-0366-2.

[17] Beveridge AJ, Wheel MA, Nash DH. The micropolar elastic behaviour of model macroscopically heterogeneous materials. Int J Solids Struct 2013;50 (1):246-55. https://doi.org/10.1016/i.ijsolstr.2012.09.023.

[18] McGregor M, Wheel MA. On the coupling number and characteristic length of micropolar media of differing topology. Proc Roy Soc A: Math, Phys Eng Sci 2014;470(2169):20140150. https://doi.org/10.1098/rspa.2014.0150.

[19] Wheel MA, Frame JC, Riches PE. Is smaller always stiffer? On size effects in supposedly generalised continua. Int J Solids Struct 2015;67-68:84-92. https://doi.org/10.1016/i.ijsolstr.2015.03.026.

[20] Nakamura S, Benedict R, Lakes R. Finite element method for orthotropic micropolar elasticity. Int J Eng Sci 1984;22(3):319-30. https://doi.org/ 10.1016/0020-7225(84)90013-2.

[21] Providas E, Kattis MA. Finite element method in plane Cosserat elasticity. Comput Struct 2002;80(27-30):2059-69. https://doi.org/10.1016/S0045-7949 (02)00262-6.

[22] Li L, Xie S. Finite element method for linear micropolar elasticity and numerical study of some scale effects phenomena in MEMS. Int J Mech Sci 2004;46(11):1571-87. https://doi.org/10.1016/i.ijmecsci.2004.10.004.

[23] Zhang H, Wang H, Liu G. Quadrilateral isoparametric finite elements for plane elastic Cosserat bodies. Acta Mech Sin 2005;21(4):388-94. https://doi.org/ 10.1007/s10409-005-0041-y.
[24] Korepanov VV, Matveenko VP, Shardakov IN. Finite element analysis of twoand three-dimensional static problems in the asymmetric theory of elasticity as a basis for the design of experiments. Acta Mech 2012;223(8):1739-50. https://doi.org/10.1007/s00707-012-0640-1.

[25] Wheel MA. A control volume-based finite element method for plane micropolar elasticity. Int J Numer Methods Eng 2008;75(8):992-1006. https://doi.org/10.1002/nme.2293. , http://doi.wiley.com/10.1002/nme.2293.

[26] Wilson EL, Ibrahimbegović A. Use of incompatible displacement modes for the calculation of element stiffnesses or stresses. Finite Elem Anal Des 1990;7 (3):229-41. https://doi.org/10.1016/0168-874X(90)90034-C.

[27] Ibrahimbegovic A, Wilson EL. A modified method of incompatible modes Commun Appl Numer Methods 1991;7:187-94. https://doi.org/10.1002/ cnm. 1630070303.

[28] Wilson EL. The static condensation algorithm. Int J Numer Methods Eng 1974;8(1):198-203. https://doi.org/10.1002/nme.1620080115.

[29] Ibrahimbegović A. Nonlinear solid mechanics: theoretical formulations and finite element solution methods. London: Springer; 2009.

[30] Wilson E, Taylor R, Doherty W, Ghaboussi J. Incompatible displacement models. In: Numerical and computer methods in structura mechanics. Elsevier; 1973. p. 43-57. https://doi.org/10.1016/B978-0-12253250-4.50008-7.

[31] Zienkiewicz OC, Taylor RL. The finite element method volume 1: the basis. Oxford: Butterworth-Heinemann; 2000.

[32] Strang G. Variational crimes in the finite element method. In: Aziz AK, editor The mathematical foundations of the finite element method with applications to partial differential equations. Academic Press; 1972. p. 689-710. https://doi. org/10.1016/B978-0-12-068650-6.50030-7.

[33] Benkemon N, Hautefeuille M, Colliat J-B, Ibrahimbegovic A. Failure of heterogeneous materials: 3D meso-scale FE models with embedded discontinuities. Int J Numer Methods Eng 2010;82:1671-88. https://doi.org $10.1002 /$ nme. 2816 .

[34] Ibrahimbegovic A, Melnyk S. Embedded discontinuity finite element method for modeling of localized failure in heterogeneous materials with structured mesh: an alternative to extended finite element method. Comput Mech 2007;40(1):149-55. https://doi.org/10.1007/s00466-006-0091-4.

[35] Kožar I, Rukavina T, Ibrahimbegović A. Method of incompatible modes overview and application. Građevinar 2018;70:19-29. https://doi.org 10.14256/ICE.2078.2017.

[36] Huang F-Y, Yan B-H, Yan J-L, Yang D-U. Bending analysis of micropolar elastic beam using a 3-D finite element method. Int J Eng Sci 2000;38:275-86. https://doi.org/10.1016/S0020-7225(99)00041-5.

[37] Malvern LE. Introduction to the mechanics of a continious medium. New Jersey: Prentice-Hall Inc; 1969.

[38] Mindlin RD, Tiersten HF. Effects of couple-stresses in linear elasticity. Arch Ration Mech Anal 1962;11(1):415-48. https://doi.org/10.1007/BF00253946.

[39] Jeffreys H. On isotropic tensors. Math Proc Cambridge Philos Soc 1973;73 (1):173-6. https://doi.org/10.1017/S0305004100047587.

[40] Lakes RS. Physical meaning of elastic constants in cosserat, void, and microstretch elasticity. Mech Mater Struct 2016;11(3):1-13. https://doi.org/ 10.2140/iomms.2016.11.217.

[41] Toupin RA. Theories of elasticity with couple-stress. Arch Ration Mech Anal 1964;17(2):85-112. https://doi.org/10.1007/BF00253050.

[42] Taylor R. FEAP - Finite Element Analysis Program; 2014. <http://www.ce. berkeley/feap>.

[43] Timoshenko S, Goodier J. Theory of elasticity. New York: McGraw-Hill; 1951.

[44] Bauer S, Schäfer M, Grammenoudis P, Tsakmakis C. Three-dimensional finite elements for large deformation micropolar elasticity. Comput Methods App Mech Eng 2010;199(41-44):2643-54. https://doi.org/10.1016/i. cma.2010.05.002.

[45] Huang F-Y, Liang K-Z. Torsional analysis of micropolar elasticity using the finite element method. Int J Eng Sci 1994;32(2):347-58. https://doi.org/ 10.1016/0020-7225(94)90014-0.

[46] Watson GN. A treatise on the theory of Bessel functions. 2nd ed. Cambridge: Cambridge University Press; 1995. 